\title{
Automated Detection of Oscillating Regions in the Solar Atmosphere
}

\author{
J. Ireland ${ }^{1} \cdot$ M.S. Marsh ${ }^{2}$ - T.A. Kucera $\left.{ }^{3}\right]$ sep \\ C.A. Young ${ }^{1}$

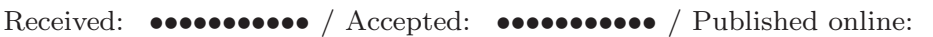

\begin{abstract}
Recently observed oscillations in the solar atmosphere have been interpreted and modeled as magnetohydrodynamic wave modes. This has allowed the estimation of parameters that are otherwise hard to derive, such as the coronal magnetic-field strength. This work crucially relies on the initial detection of the oscillations, which is commonly done manually. The volume of Solar Dynamics Observatory (SDO) data will make manual detection inefficient for detecting all of the oscillating regions. An algorithm is presented which automates the detection of areas of the solar atmosphere that support spatially extended oscillations. The algorithm identifies areas in the solar atmosphere whose oscillation content is described by a single, dominant oscillation within a user-defined frequency range. The method is based on Bayesian spectral analysis of time-series and image filtering. A Bayesian approach sidesteps the need for an a-priori noise estimate to calculate rejection criteria for the observed signal, and it also provides estimates of oscillation frequency, amplitude and noise, and the error in all these quantities, in a self-consistent way. The algorithm also introduces the notion of quality measures to those regions for which a positive detection is claimed, allowing simple post-detection discrimination by the user. The algorithm is demonstrated on two Transition Region and Coronal Explorer (TRACE) datasets, and comments regarding its suitability for oscillation detection in SDO are made.
\end{abstract}

Keywords: Sun: active region, Sun: magnetic field

\section{Introduction}

The Solar and Heliospheric Observatory (SOHO) and TRACE missions established that the solar corona supports observable oscillations. The extensive literature on the theory of oscillations in coronal flux tubes has been used

\footnotetext{
1 ADNET Systems, Inc., NASA's Goddard Spaceflight Center, Mail Code 671.1, Greenbelt, MD 20771, USA. (e-mail: jack.ireland@nasa.gov)

2 Jeremiah Horrocks Institute, University of Central

Lancashire, Preston, PR1 2HE, UK.

3 NASA's Goddard Spaceflight Center, Mail Code 671.1, Greenbelt, MD 20771, USA.
} 
in conjunction with observationally derived parameters to deduce conditions in the corona. The study of these oscillations is called coronal seismology (in analogy to terrestrial seismology). Their general features, as determined by current analyses, and their implications for the physics of the corona are summarized briefly below; more detailed reviews can be found in De Moortel (2005) and Nakariakov and Verwichte (2005). Quasi-periodic oscillations, interpreted as propagating slow waves have been detected in coronal plumes SOHO/Ultraviolet Coronal Spectrograph (UVCS: Ofman et al., 1997); SOHO/Extreme Ultraviolet Imaging Telescope (EIT: Deforest and Gurman, 1998). A similar phenomenon (at higher frequency) has also been detected at the base of coronal loops (Berghmans and Clette 1999, Nightingale, Aschwanden, and Hurlburt, 1999: De Moortel, Ireland, and Walsh. 2000, Robbrecht et al., 2001). They are interpreted as propagating slow waves since they travel at approximately the sound speed and are seen as intensity (and therefore density) variations propagating and decaying away from the base of the flux tube through the corona. The observed periodicities fall into distinct ranges around three and five minutes (De Moortel et al., 2002), suggesting that the oscillations are due to different connectivity to either sunspot or transitionregion moss magnetic field respectively. Coronal seismological applications for these oscillations include determination of the connectivity of the photosphere to the corona (de Pontieu, Erdélyi, and De Moortel, 2005) as well as deriving information on the coronal heating function since the wave observables (e.g., period and damping length) are strongly dependent on the thermal conditions of the corona (De Moortel and Hood, 2003, De Moortel and Hood, 2004).

All of these studies have in common that the oscillating feature was discovered by a manual examination of data in regions known (or suspected) to contain oscillating material. Although a successful strategy for detecting oscillations, it is clearly impractical for ever-increasing rates of data acquisition. For example, the SDO mission is acquiring i1.4 Tb of science data per day, around 1000 times the data rate of $\mathrm{SOHO}$. Much of this data will be taken at cadences and spatial resolutions comparable to the best that TRACE can produce, the key difference from TRACE being that SDO will provide a near continuous, full disk coverage of the Sun in multiple wavelengths simultaneously. Given the diagnostic possibilities of these waves, and the large amount of data that will become available, an automated detection algorithm is necessary. There are at least five published algorithms, which are briefly described below.

Nakariakov and King (2007) use a thresholded fast Fourier Transform to find locations in TRACE data that may support an oscillatory signal. The threshold level is defined as three-four times the average FFT power; if the maximum FFT power is above this level then the frequency at which that power occurs is assumed to be real. Since this method relies on the FFT, it is very fast. The definition of the threshold is chosen to speed up the algorithm in comparison to calculating a threshold; for example, implementing the randomization method of Linnell-Nemec and Nemec (1985) requires at least an estimated 150 times more FFT calculations. Linnell-Nemec and Nemec (1985) establish frequency acceptance/rejection criteria based on probabilistic arguments whereas Nakariakov and King (2007) use essentially empirically derived arguments to establish the threshold. Further, identification of contiguous groups of pixels 
that might form an oscillatory region is carried out by manual inspection. To aid identification, the authors assume a null hypothesis that the entire timeseries consists solely of Gaussian distributed noise, and so the chance that any two neigboring pixels contain the same frequency is small. Hence, if two or more neighboring pixels do satsisfy the selection criteria, then it is likely they are physically connected. These assumptions ignore two effects: firstly, neighboring pixels are not statistically independent due to the point spread function of the instrument. Secondly, the Sun is observed to have physically connected structures, such as loops, that exist over many pixels in at least one direction, and so by continuity, one can expect that neighboring pixels do influence each other. Hence the assumption that neighboring pixels are independent of each other is an over-simplification of the nature of the image. The authors suggest that the algorithm be used to identify regions in the data worthy of further study, although there is no quoted method of automated region identification.

De Moortel and McAteer (2004) describe an automated oscillation detection algorithm based on the wavelet analysis routines of Torrence and Compo (1998) and the analysis procedure of Ireland et al. (1999). The algorithm finds significant wave packets ranging from single to multiple wave cycles in duration, by a wavelet power/confidence level comparison against the null hypothesis that a given time-series is Gaussian distributed noise. Shorter duration detections are rejected. Contiguous regions of multi-cycle duration wave packets are found in the data, but are identified and isolated manually by inspection.

Sych and Nakariakov (2008) base their detection algorithm on pixelized wavelet filtering (PWF) of a three-dimensional data cube $(x, y, t)$. This too is based on the wavelet analysis routines of Torrence and Compo (1998), but has a more complex treatment of the resultant wavelet spectrum. Regions of interest are found by first calculating a variance map (Grechnev, 2003) of the signal; regions with high variance are candidate oscillatory regions (note that this must also imply the removal of a background trend in order for the variance to measure an oscillatory signal, and not the trend). Wavelet spectra are calculated for those pixels, and only the "significant" pixels are retained (what constitutes a significant signal is not stated explicitly). The routine analyzes further (than De Moortel and McAteer, 2004) the temporal evolution of the oscillation and so can differentiate between standing and traveling waves.

The algorithm presented by McIntosh, de Pontieu, and Tomczyk (2008) can also differentiate between standing and traveling waves. The algorithm begins by Fourier transforming the entire data cube, performing cross correlations with neighboring pixels in narrow frequency bands, and filtering the results (by thresholding on various quantities, such as eliminating areas where the relative error in the calculated phase speed is large) to determine groups of pixels that are highly correlated in both time and space. This correlation technique allows the discrimination of standing and traveling waves, and the calculation of other parameters such as the phase speed and the propagation angle. There is an implicit null hypothesis in the algorithm: at one stage, only time-series with a high coherence are accepted for further analysis. The null hypothesis here is that the candidate time-series is pure noise, with the additional assumption that pairs of noisy time-series have low coherence, and so can be rejected. However, this is 
not strictly true; Chatfield (1996) shows an example of two different noisy timeseries that have a perfect coherence over all spectral frequencies because both time-series are generated from the same noise process. Highly coherent patches of non-oscillatory material are probably filtered out in the next stage of the algorithm, where regions with a poorly determined phase speed (large error) are discarded; this, however, has not been explicitly tested (S. W. McIntosh, private communication, 2008).

It is clear that there is an increasing amount of effort in finding oscillatory regions and identifying waves in the solar atmosphere. All the above algorithms show promising results and avenues for further work. The algorithm presented here seeks to find regions in the data which support oscillations via Bayesian time-series analysis. This involves calculating explicitly the probability that a time series supports an oscillation of a given frequency. This is in distinction to the methods above, which rely on statements about null hypotheses in order to determine if an oscillation is present. Section 2 introduces Bayes' Theorem and an application of it to time-series analysis. Section 3 describes a detection algorithm based on the results of Section 2, whilst Section 4 describes the application of this algorithm to some example datasets from the TRACE mission. Finally, Section 6 discussions some further applications of Bayesian time-series analysis and automated EUV detection algorithms.

\section{Bayesian Time-Series Analysis}

Denoting by $p(a \mid b)$ the conditional probability that proposition $a$ is true, given that proposition $b$ is true, Bayes' theorem is

$$
p(H \mid D, I)=\frac{p(H \mid I) p(D \mid H, I)}{p(D \mid I)}
$$

where $H$ is the hypothesis to be tested, $D$ is the observation, and $I$ is any applicable prior information that we have before making the observation (Bayes, 1763). The left hand side $p(H \mid D, I)$ is called the posterior probability of the hypothesis, given the data and the prior information, and it encapsulates the available knowledge about the hypothesis. The quantity $p(H \mid I)$ is called the prior distribution and represents what we know about $H$ prior to any data collection. Often a prior describes a probability distribution of likely parameter values. The sampling distribution or likelihood $(p(D \mid H, I))$ represents the likelihood of the data given the hypothesis (as well as any prior information). The quantity $p(D \mid I)$ is the prior probability of the data; it is absorbed into a normalization constant, and does not affect the following analysis for a given model. Equation (1D) is very general and is not restricted to the mathematical equations: any logical proposition can be treated in a Bayesian context (Jaynes, 2003, Gregory, 2005).

\subsection{Signal Containing a Single Frequency}

For the purposes of detecting oscillations in solar time-series $d\left(t_{i}\right)$, the linear model

$$
d\left(t_{i}\right)=b_{1} \cos \omega t_{i}+b_{2} \sin \left(\omega t_{i}\right)+x_{i}
$$


is used. This assumes that all time-series are modeled as a single oscillation plus Gaussian distributed noise. Under some simplifying assumptions $(N>>1$ and that there are no low-frequency oscillations present), Jaynes (1987), Bretthorst (1988), and Ó Ruanaidh and Fitzgerald (1996) derive an expression for the probability that the time-series contains an oscillation of frequency $\omega$ for the model oscillation above, that is,

$$
p(\omega \mid \mathbf{d}, I) \propto\left[1-\frac{2 C(\omega)}{n \rho^{2}}\right]^{(2-n) / 2}
$$

where $\rho=1 / n \sum_{j=1}^{n} d_{j}^{2}$ and

$$
C(\omega)=\frac{1}{N}\left|\sum_{j}^{n} d_{j} e^{i \omega t_{j}}\right|^{2}
$$

is the Schuster periodogram (Schuster, 1898). The full details of the derivation of Equation (3) are contained in the appendix. This is a probability measure that a given frequency is present in the data that does not require explicit knowledge of the Gaussian noise present. Equation (3) can analyze unevenly sampled data due to its use of the Schuster periodogram. If the analysis frequencies are

$$
\omega_{p}=2 \pi p / n, 0 \leq p \leq n / 2-1,
$$

Equation (4) is proportional to the power of the fast Fourier transform (FFT) of the data (Cooley and Tukey, 1965, Chatfield, 1996). The speed of the FFT transform can be exploited for data which are evenly or close to evenly spaced.

\subsection{Example Analysis}

Figure 1demonstrates the application of the above equations to the analysis of a single time-series. The true oscillation is an evenly sampled simple cosine at frequency $f=3.33 \mathrm{mHz}$ (100 data points at a cadence of 26.4 seconds) with unit amplitude. The fake data is this oscillation corrupted by Gaussian distributed noise with deviation of $\sigma=1$, a signal-to-noise ratio of 1 (Figure प(a)). The probability function, Equation 3, is highly peaked close to the true frequency and the probability that the signal contains a frequency within the range 3.0$3.5 \mathrm{mHz}$ exceeds $99 \%$ (Figure 1(b)). Figures 1(c,d) show the measured basis function amplitudes and Gaussian deviation estimate respectively as a function of frequency. Both lie close to their true values at the true frequency, as indicated by the solid vertical line. The quoted values shown in each plot (Figure 1 (b,c,d)) are found at $\omega=\hat{\omega}$, the most probable frequency. An error estimate is given by the standard deviation

$$
[\operatorname{sd}(\hat{\phi})]^{2}=\sum_{i=1}^{N_{\omega}} p\left(\omega_{i}\right)\left[\phi\left(\omega_{i}\right)-\phi(\hat{\omega})\right]^{2}
$$


(a) time series

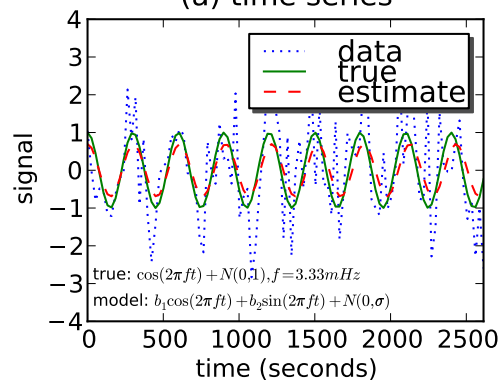

(c)

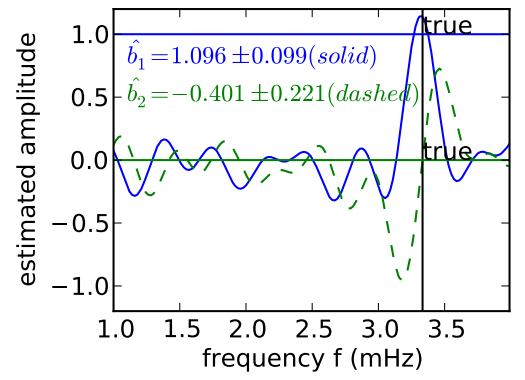

(b) probability

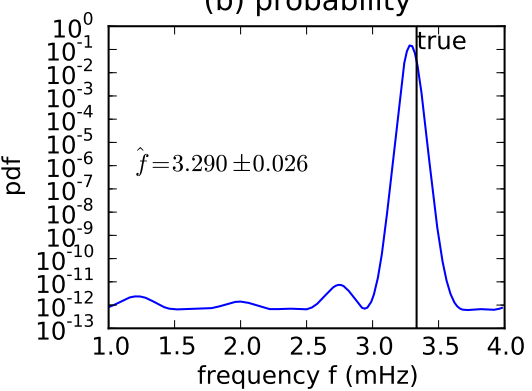

(d)

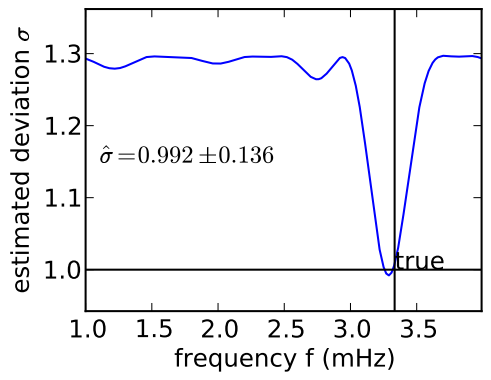

Figure 1. An example analysis using the Bayesian approach of Section 2 Panel (a) shows the true oscillation, the observed (noisy) data, and the estimate. Panel (b) shows the probabilitydensity function arising from the observed data. Panel (c) shows the estimated amplitudes as a function of frequency $[f]$, and panel $(d)$ shows the estimated noise as a function of frequency. Final quantity estimates and an estimated error are shown also. See Section 2 for more detail.

where $\phi(\omega)$ stands for the analyzed frequency range, the distribution of amplitudes (found from Equation (13) - see appendix) and the distribution of $\sigma$ (found from Equation (15) - see appendix) as a function of the analysis frequency set $\omega_{i}, 1 \leq i \leq N_{\omega}$ (such as the Fourier set, Equation (50)).

These estimates of frequency probability, oscillation amplitude and Gaussian noise deviation have all been obtained without explicitly performing a leastsquares fit, or with no special a-priori knowledge of the noise in the signal other than the assumption of a Gaussian distribution.

The peakedness of the Bayesian probability-density function immediately suggests that a search for portions of the $\{\omega\}$ parameter space that contain most of the probability are the values that are of greatest interest. In addition, prior knowledge from previous studies of solar atmospheric oscillations suggests which regions of the parameter space are of interest. These two observations are combined below to take the first steps towards creating an automated oscillation detection algorithm which can identify oscillation regions and return useful information, such as their amplitude. 


\section{Detection using Bayesian Spectral Analysis}

\subsection{Data}

The data that we will use are three dimensional datacubes $(x, y, t)$, with the $x$ and $y$ locations referring to spatial locations (pixels) on the Sun, and $t$, time. time-series are formed by choosing a particular location on the Sun and extracting a one-dimensional time-series (occasionally super-pixels formed by the sum of neighbouring pixels are used to increase the signal-to- noise ratio).

\subsection{Probability and Frequency Bands}

In order to use Equation (3) to detect oscillations, further assumptions must be made. The algorithm assumes that the range of possible frequencies is limited to that spanned by the Fast Fourier Transform applied to a time-series of similar length. This permits us to normalize the probablity density function over a fixed range of frequencies. We are commonly interested in detecting oscillations in given frequency bands (say the three or five minute oscillation frequency bands), and so we calculate the probability that the oscillation in a given pixel lies within the range $\left[\omega_{1}, \omega_{2}\right]$, that is,

$$
p_{\omega_{1}, \omega_{2}}=\int_{\omega_{1}}^{\omega_{2}} p(\omega \mid D, I) \mathrm{d} \omega
$$

at every point in the image. Large values of $p_{\omega_{1}, \omega_{2}}$ indicate that the true oscillation frequency is very likely to be within the range $\left[\omega_{1}, \omega_{2}\right]$.

The Bayesian formulation handily yields both computational and logical advantage over other frequentist approaches for oscillating-pixel detection. The prime derived data product for each pixel is a probability distribution describing the probability of a given frequency in the data and so there is no need to perform secondary, often computationally expensive calculations, such as randomization tests (Linnell-Nemec and Nemec, 1985, O'Shea et al., 2001) to assess the probability that the frequency is present or not. Frequentist-based approaches to detecting oscillations rely on calculating the expectated value of the Fourier transform power at a given confidence level, assuming that the observed data arises from the null hypothesis that the time-series is pure noise. If the Fourier transform power of the observed time-series exceeds the expectation value, then the null hypothesis is rejected at that confidence level. This, however, strictly cannot be used to imply the presence of an oscillation; we have merely rejected the null hypothesis. In comparison, Equation (7) directly calculates the probability that the frequency of oscillation lies in the range $\left[\omega_{1}, \omega_{2}\right]$, under the modeling assumptions of Section 2 .

\subsection{An Algorithm}

Figure 2 describes the general-purpose algorithm used to find oscillating spatial locations in the data cube. Step 2 relies on instrument calibration routines provided by instrument teams, and on standard solar de-rotation routines, both 


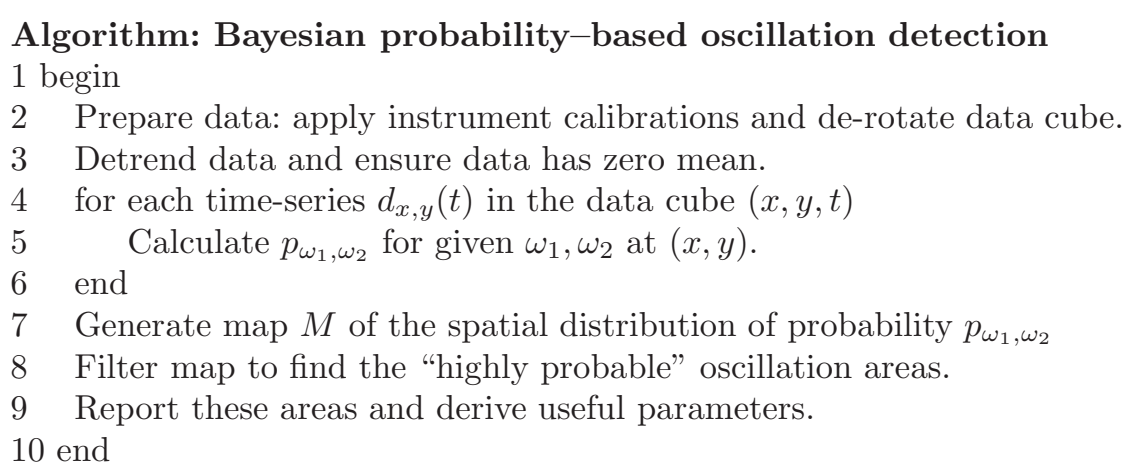

Figure 2. Pseudo-code algorithm to find oscillating locations in $(x, y, t)$ data cubes.

provided in the IDL/Solarsoft package. Time-series de-trending (Step 3) is necessary for two reasons. The first is dictated by our choice of oscillation model, Equation (2). This model assumes that the time series contains a single oscillation only, and no other features. (It is certainly possible to define other models that do contain a background trend, or multiple frequencies, and calculate probability density functions for those time-series. However, these more sophisticated analyses are not necessary for us to make progress in the current application of locating oscillating material). Secondly, strong background trends can pollute the Fourier power spectrum with spectral power unrelated to the oscillation. This can lead to the mis-identification of peaks in the power spectrum as oscillatory when they are not. It should be noted that the influence of background trend on locating oscillations of a given frequency will influence all proposed automation algorithms.

De-trending in Step 3 is accomplished by subtracting a running average of the time-series (a window of size $R$ seconds is slid across the time-series and the running average of the data lying entirely within that window is calculated). Since previous experience has told us that there are oscillations of interest that have periods less than 500 seconds, it seems to us that 500 seconds is a reasonable to chose for these data (Aschwanden et al. 2002). Timescales longer than $R$ can be considered to be associated with the background trend. Timescales shorter than $R$ potentially support an oscillation. Fourier power spectra of smooth timeseries that have had their background trend subtracted have extremely low power at frequencies less than that corresponding to $R$. Therefore, these low frequencies corresponding to the background trend have an extremely low probability and are not selected.

Step 5 finds the probability that the time-series has a single frequency in the range $\left[\omega_{1}, \omega_{2}\right]$. There are several ways of defining $\left[\omega_{1}, \omega_{2}\right]$. In the work below the algorithm begins by first finding the location $\omega_{\max }$ of the highest peak in the probability distribution function (PDF) $p(\omega \mid D, I)$. If $\left[\Omega_{1} \leq \omega_{\max } \leq \Omega_{2}\right]$, where $\left[\Omega_{1}, \Omega_{2}\right]$ are defined by the user (a frequency filter), then the algorithm proceeds by stepping away from the peak to find the nearest turning points in the PDF, 
Jul. 1

(a)

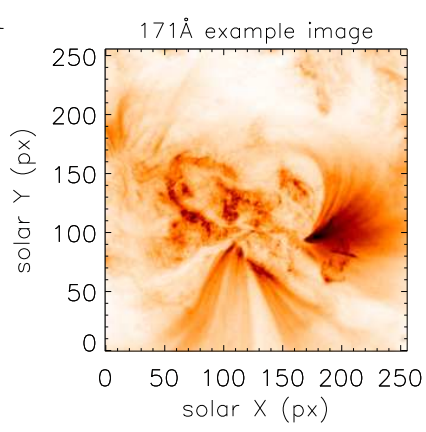

(b)

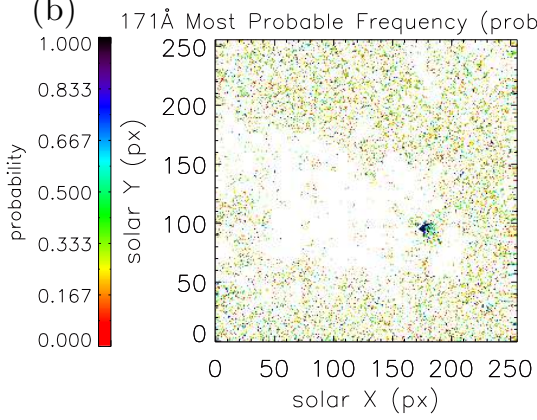

(c)

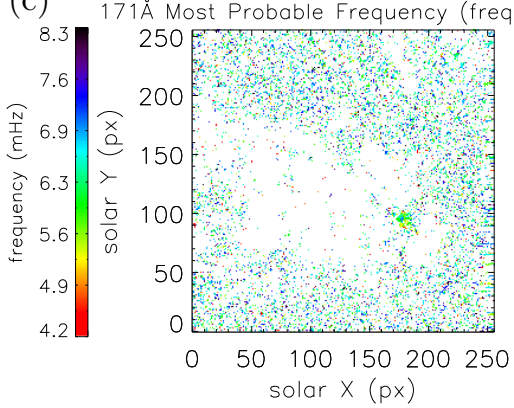

Jul. 14

(d)

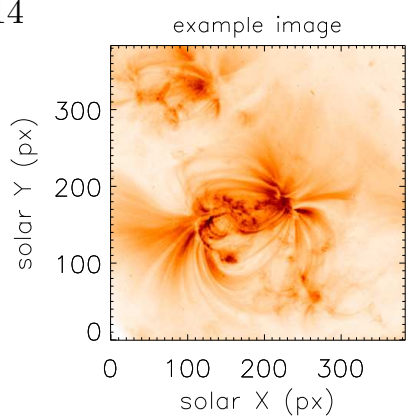

(e) Most Probable Frequency (probability)

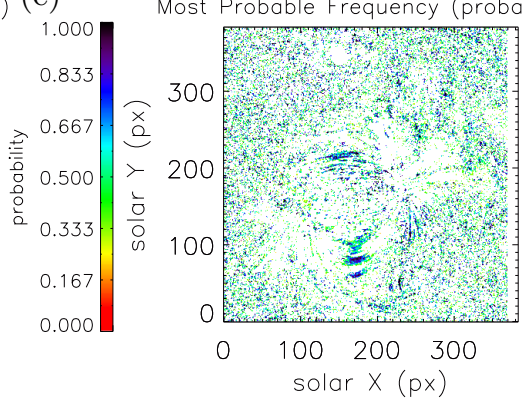

(f) Most Probable Frequency (frequency)

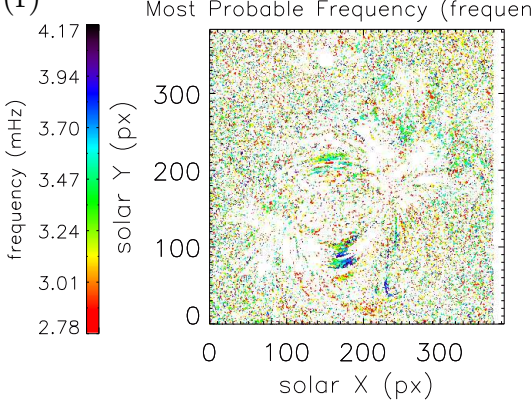

Figure 3. Example image data and probability and frequency maps. Panels a, b, and c refer to TRACE $171 \AA$ data taken on 1st July 1998 and analyzed for oscillations in the three-minute frequency band $4.17 \leq f_{\max } \leq 8.33 \mathrm{mHz}$ ), and panels $\mathrm{d}$, e and $\mathrm{f}$ refer to TRACE $171 \AA$ data taken on 14 July 1998 and analyzed for oscillations in the five-minute frequency band $\left(2.78 \leq f_{\max } \leq 4.17 \mathrm{mHz}\right)$.

located at $\left[\omega_{1}, \omega_{2}\right]$. The probability $\left[p_{\omega_{1}, \omega_{2}}\right]$ is then calculated. This creates the probability map $M$ of Step 7.

Figure 3(a,b,c) shows some example data, and its analysis up to Step 7. Two datasets are examined, both in the TRACE $171 \AA$ wave-band. The first dataset consists of 391 observations with a mean 31s cadence taken on 1 July 1998 12:03:10 UT - 15:28:52 UT. The resulting probability map and its concomitant frequency map are shown in Figures $3(b, c)$ respectively. There appears to be 
a single large area that appears to contain a significant oscillation at differing frequencies and probabilities.

Cleanly extracting oscillating features is much more difficult for the second example dataset shows in Figures $3(d, e, f)$. Here there are many groups of pixels having similar frequencies and high probability close to each other. In addition, there are many pixels that have high probability scattered all over the field of view, further complicating the task of extracting a single group. The eye is very good at picking out such groups, and the algorithm attempts to mimic some of this behavior. The detection algorithm attempts to mimic this process by examining the local spatial distribution of probability. Firstly, it is assumed that nearby pixels of high probability are physically related, even if their frequencies may be different. However, these groupings may not be contiguous due to noise, for example. This may be overcome by smoothing the probability map at different lengthscales, and flagging those areas which exceed a certain threshold. This has the effect of the allowing discontinuous areas of high probability to merge, in a similar way to how the eye may integrate physically "close" oscillating areas into one group. More formally, Step 8 implements

1. for $m$ an integer in the range $\left[L_{1}, L_{2}\right]$ :

a) Smooth the probability map $[M]$ with a Gaussian filter of width $m$.

b) Generate a mask $\left[Z_{m}\right]$ locating all the areas in the smoothed map that have a smoothed probability at or over 0.5

The lengthscales $m$ allow the user to examine the average spatial probability structure of the data on multiple lengthscales. This procedure is meant to mimic the way the eye examines such probability maps, where the eye finds it easy to integrate over neighbouring high probability features. The upper lengthscale $\left[L_{2}\right]$ describes the maximum distance that two pixels are assumed to be potentially physically related to each other. Smaller maximum values of $m$ cause the smoothed probability masks to break up more, whereas larger values can cause the creation of groups of pixels which are essentially unrelated. The masks $\left[Z_{m}\right]$ found in step $1 \mathrm{~b}$ denote the areas that are more likely than not to support contiguous oscillations over the lengthscale $m$. The net effect of step 1 is to implement a simple multi-scale analysis of the spatial probability structure.

2. Add all the masks $\left[Z_{m}\right]$ together. This is the mask $-Z-$ of all the candidate pixel groups that may support a significant oscillations.

3. Remove from $Z$ all the candidate pixel groups that form a group smaller than $g$ pixels. Nominally, we set a minimum group size $g=L_{2}^{2}$; that is, we look for groups of pixels that are connected over the largest distance set in the multi-scale analysis of step 1.

4. For a candidate group of area $A$, remove that group from $Z$ if it has more than $h A$ zero-probability pixels, $0 \leq h \leq 1$. The remaining pixel groups are considered to be regions in the solar atmosphere that support oscillations in the range $\Omega_{1}, \Omega_{2}$.

In the results below, $L_{1}=1$ and $L_{2}=4, g=16$; these are chosen in order to allow smaller width strands to be found in the data. The last part of Step 
Table 1. Quantities reported for a contiguous group $[G]$ of pixels found by the detection algorithm of Figure 2

\begin{tabular}{clc} 
quantity & definition & units \\
\hline $\bar{f}$ & average frequency in a group $G$ & $\mathrm{mHz}$ \\
$\sigma_{f}$ & standard deviation of frequency & $\mathrm{mHz}$ \\
$A$ & area of $G$ with a non-zero probability & area $(\mathrm{px})$ \\
$A_{0.95}$ & area of $G$ with $p_{\omega_{1}, \omega_{2}} \geq 0.95$ & area $(\mathrm{px})$ \\
$F$ & $A /($ area of group $G)$ & dimensionless \\
$\bar{p}$ & average probability of all pixels having a non-zero probability & dimensionless \\
$Q$ & $F \bar{p}($ quality of the group $G)$ & dimensionless \\
$E$ & $A Q($ the equivalent area of the group $G)$ & area $(\mathrm{px})$
\end{tabular}

8 acknowledges that in performing the probability-map smoothing, pixels that contain no oscillation in the range $\Omega_{1}, \Omega_{2}$ can be swept into a candidate pixel group $A$, and this must have a limit lest the candidate pixel group have too many lacunae. The final step (Step 9, Figur(2) is to report properties of the remaining pixel groups such as the average frequency $[\bar{f}]$ and the standard deviation $\left[\sigma_{f}\right]$ of the frequency. The full table of reported results is given in Table 1 In any given analysis, the regions found result from a series of filters and thresholds made by the user. However, not all of the regions that survive this process are necessarily equivalent, they have merely satisfied some set of criteria. These quantities attempt to measure differences between the surviving oscillatory regions, and allow the user to further discriminate them at their discretion. Clearly the size [area $A$ ] of the pixel group is important, and the number of highly probable pixels $\left[A_{0.95}\right]$. The quantity $F$ measures how complete the group is, and $\bar{p}$ measures the average probability that the average oscillation frequency of $G$ is indeed between $\Omega_{1}, \Omega_{2}$. The quality $[Q]$ is an average probability for the entire group, and the equivalent area $[E]$ is the area that the group would have if it were entirely complete (no lacunae) and every oscillation detected in it had $p_{\omega_{1}, \omega_{2}}=1$. These measures, along with maps of the detected regions showing frequency, amplitude, and noise estimates (along with error estimates to each of these quantities), are the final derived products of this automated detection analysis.

\section{Results}

Data was analyzed from two TRACE observing sequences. Data was prepared using standard IDL/Solarsoft TRACE routines (TRACE_PREP with the keywords /wave2point, /unspike, /destreak, /deripple, /norm, /float switched on) and derotated using IDL/Solarsoft SHIFT_XY to calculate the motion of the observed piece of Sun, and cubic two-dimensional interpolation to shift subsequent images back to the location of the first one. In addition, a strip of data of width ten pixels at the extreme right of the image is removed, as this is the location of severe image distortion due to image derotation. Further, all time-series are detrended by removing a running average taken over a $R=500$ second timescale (see Step 3, Figure 2). In the following results, signal-to-noise 
ratio $(\mathrm{SNR})$ is calculated as the estimated amplitude $\sqrt{{\hat{b_{1}^{2}}}^{2}+{\hat{b_{2}}}^{2}}$ (see Equation (2)) divided by the Gaussian noise estimate $\hat{\sigma}$.

\subsection{July 1998}

The original data was taken on 1 July 1998 12:30:01 - 14:24:41 UT with an average cadence of 31 seconds ( 220 samples) and an image size of $512 \times 512$ pixels of equivalent size $0.5^{\prime \prime}$. Data are also $2 \times 2$ summed in space to increase signal-to-noise ratio (this has the consequent effect of reducing the algorithm run time). The results of searching in a wideband three-minute range $(120-240$ seconds, or $4.17-6.33 \mathrm{mHz})$ are shown in Figures $4(171 \AA)$ and 5 (195 $\AA)$ and in Table 2(a,b). Most of the field of view does not contain material oscillating in the analysis frequency range. Only one group of pixels survives the filtering process, at the base of a coronal-loop fan (Figure 6). However, it is interesting to note that there are areas of the probability maps Figures 4 (b), 5(b) that have effectively zero probability of supporting an oscillation in the frequency band, whilst others have a non-zero and low-probability. We speculate that with the improved SNR of SDO, many of these low probability oscillations will become much more likely, leading to the detection of many more spatially distributed signals in the data.

The single oscillating pixel group found (Figure 6) is in the same location as that identified manually by King et al. (2003) and automatically by McIntosh, de Pontieu, and Tomczyk (2008). The region is distinctly different from others in the data. Figures 4 (f) and 5 (f) show that the detected oscillations in this pixel group has a fairly low estimated signal-to-noise ratio. The estimated error in the frequency decreases with increasing SNR, as expected.

The results of searching in the five-minute frequency band (240-360 seconds, or $2.78-4.17 \mathrm{mHz})$ are shown in Figures $7(171 \AA)$ and $8(195 \AA)$ and in Table 2(c,d). The probability maps Figures 4(b), 5(b) look very different in this frequency range, with low-probability oscillations scattered across the field of view. It is noticeable, however, that the higher-probability oscillations are concentrated in the core of the active region, in the regions that appeared empty in Figures 4(b), 5(b). The algorithm does qualify some pixel groups as supporting oscillations. King et al. (2003) do not claim any detections in these areas (although it is not clear if they looked), and McIntosh, de Pontieu, and Tomczyk (2008) find only one coherent pixel group in the same general region as those found here. Some of the regions do overlap, suggesting co-temporal and cospatial propagation of oscillations in two different layers of the atmosphere. In the image, the areas in question resemble TRACE moss (Berger et al., 1999. de Pontieu et al., 1999. Fletcher and de Pontieu, 1999). However the presence of groups of oscillations at multiple temperatures argues that the algorithm has found examples of leakage of five minute oscillations from lower down in the atmosphere to the upper layers, as described by de Pontieu, Erdélyi, and James (2004) and de Pontieu, Erdélyi, and De Moortel (2005) and references therein.

Finally, the plotted points in Figures 7 (f), 8(f) appear to lie on horizontal lines across the plot, which are simply the analysis frequencies. The foregoing analysis can be done with many more frequencies (see Section 2.1), at the expense of using 
Table 2. Quantities reported (see Table 1 for the automatically detected regions found in 1 July 1998 TRACE $171 \AA$ and $195 \AA$ data - see Figures 4 [5 7 and 8 for maps and plots of the detected regions listed in (a), (b), (c) and (d) respectively above. The quantities $Q$ and $E$ are also listed with their rank $[r]$ when compared to all other regions found in the same dataset.

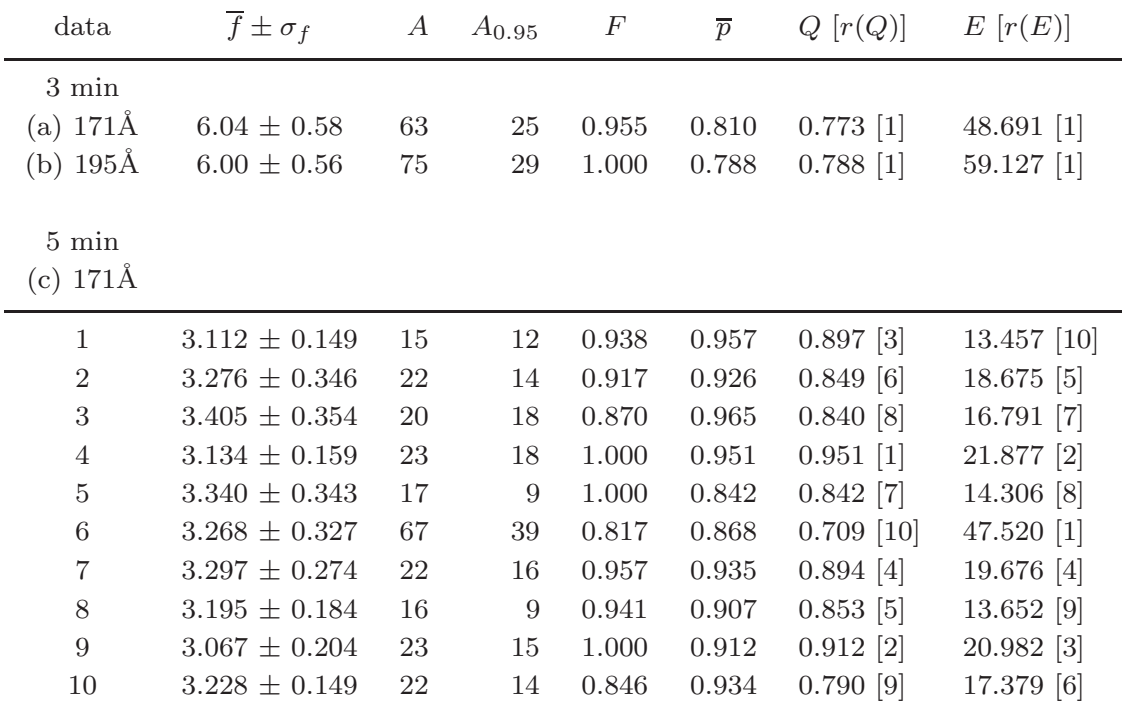

(d) $195 \AA$

\begin{tabular}{lllrllll}
\hline 1 & $3.164 \pm 0.214$ & 31 & 15 & 0.912 & 0.863 & $0.787[6]$ & $24.402[5]$ \\
2 & $3.209 \pm 0.334$ & 31 & 18 & 0.886 & 0.904 & $0.801[5]$ & $24.833[4]$ \\
3 & $3.342 \pm 0.395$ & 75 & 42 & 0.852 & 0.878 & $0.748[9]$ & $56.099[1]$ \\
4 & $3.166 \pm 0.254$ & 16 & 6 & 1.000 & 0.840 & $0.840[3]$ & $13.433[8]$ \\
5 & $3.240 \pm 0.286$ & 41 & 25 & 0.911 & 0.895 & $0.816[4]$ & $33.451[2]$ \\
6 & $3.471 \pm 0.289$ & 19 & 11 & 1.000 & 0.911 & $0.911[1]$ & $17.305[6]$ \\
7 & $3.295 \pm 0.277$ & 17 & 12 & 0.850 & 0.914 & $0.777[8]$ & $13.214[9]$ \\
8 & $2.998 \pm 0.133$ & 17 & 11 & 0.944 & 0.910 & $0.859[2]$ & $14.606[7]$ \\
9 & $3.200 \pm 0.174$ & 34 & 21 & 0.895 & 0.870 & $0.778[7]$ & $26.452[3]$
\end{tabular}

the FFT to perform the analysis. This would slow performance, but would lead to a more precise knowledge of the frequencies present. Since we are primarily interested in detecting the presence of frequencies in a wide frequency band, the precision of each frequency detected is not as important as their detection in as little time as possible.

\subsection{July 1998}

The original data was taken on 14 July 1998 12:45:19 - 13:42:44 UT with an average cadence of 73 seconds (sixty samples) and an image size of $512 \times 512$ pixels of equivalent size $0.5^{\prime \prime}$. Data are also $2 \times 2$ summed in space to increase signal-to-noise ratio. TRACE was observing NOAA AR 8270 when a GOES class M4.6 flare occurred at around 12:55 UT. 
(a)

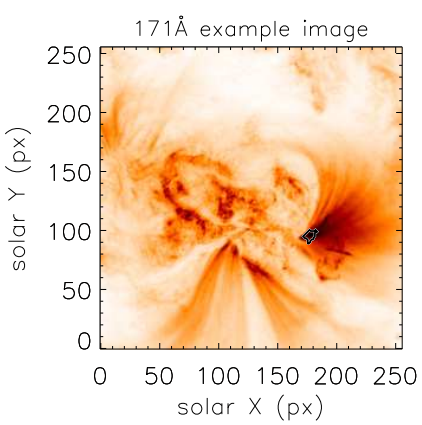

(c)

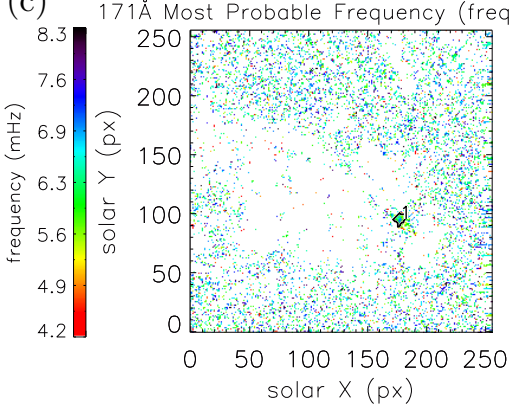

(e)

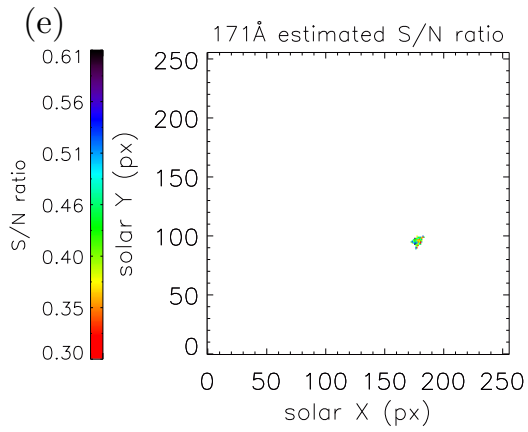

(b)
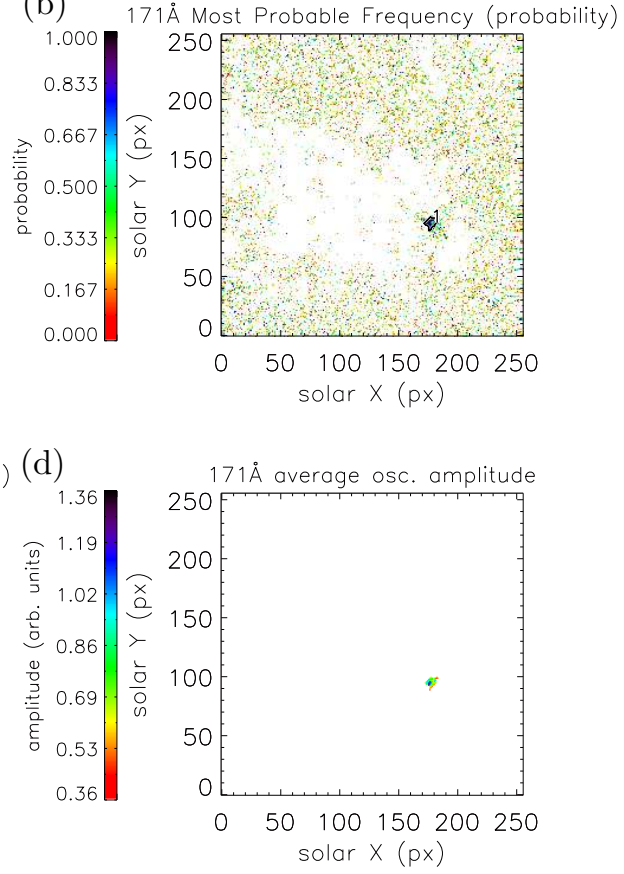

(f)

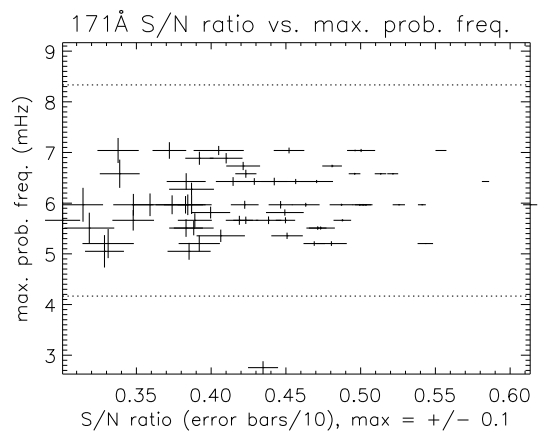

Figure 4. Example image data and results from the analysis algorithm for TRACE $171 \AA$ data taken on 1 July 1998 (panel (a)). Panel (b) is the probability that a given pixel supports an oscillation within the frequency band indicated in panel (c), in this case, a three-minute oscillation frequency band. Panel (c) shows the frquency supported at those pixels. Also indicated in panels $(\mathrm{a}-\mathrm{c})$ are the detected oscillation regions. Note that the region agrees with that found manually by King et al. (2003) on the following day. The oscillation region is found at the base of a coronal loop structure, as has been found by many authors (De Moortel et al. 2002). Panel (d) shows a map of estimated amplitude for the detected regions, whilst panel (e) shows a map of the signal to noise ratio. Finally, panel (f) shows a panel of the detected frequency as a function of the signal to noise ratio. The error bars on the abscissa values are shown at one-tenth of their actual size in order to better show the lower error values at higher signal-to-noise ratio. Table 2 (a) shows the values for the detected region for the parameters listed in Table 1 
(a)

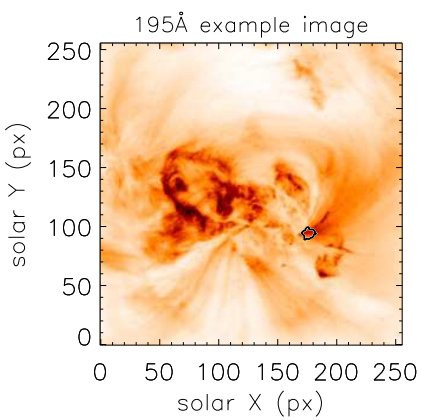

(c)

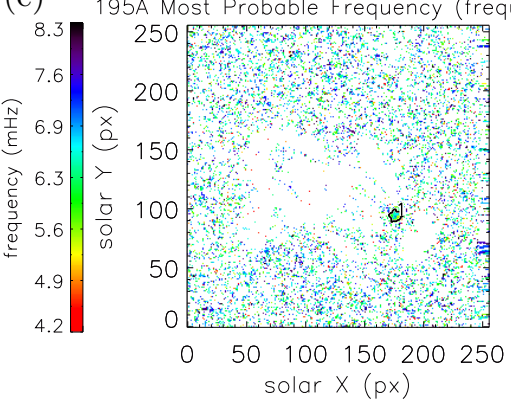

(e)

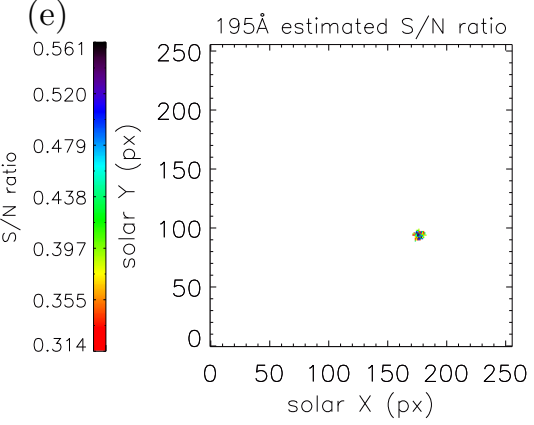

(b) 195: Most Probable Frequency (probability)

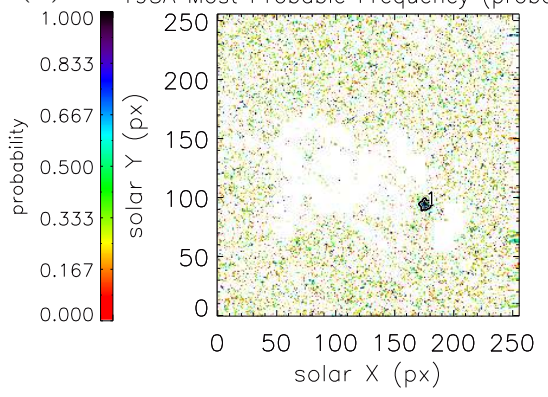

(d)

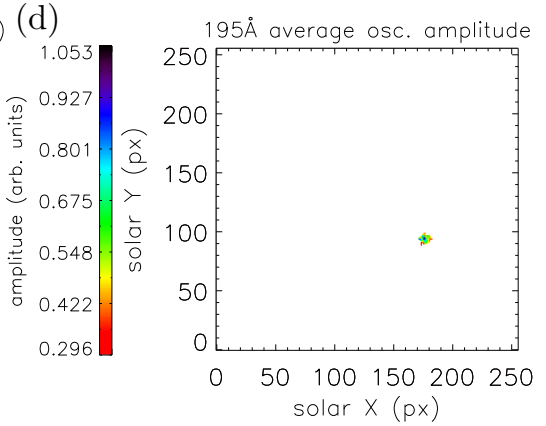

(f)

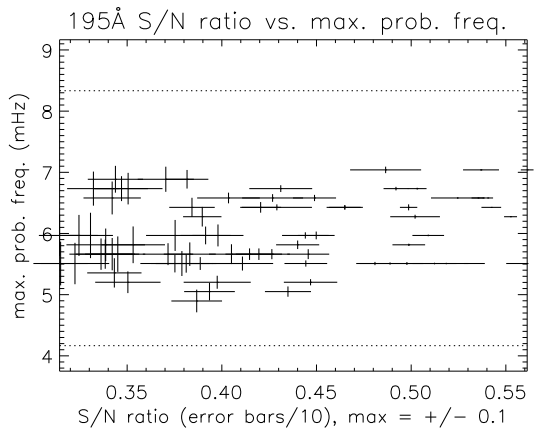

Figure 5. Example image data and results from the analysis algorithm for TRACE $195 \AA$ data taken on 1 July 1998 (panel (a)). Panel (b) is the probability that a given pixel supports an oscillation within the frequency band indicated in panel (c), in this case, a five-minute oscillation frequency band. Note that the region agrees with that found manually by King et al. (2003) on the following day. Panels $(\mathrm{d}-\mathrm{f})$ show similar maps to those shown and described in Figure 4 Table 2 b) shows the values for the detected region for the parameters listed in Table 1

A movie of the analyzed data cube shows that the first eight samples show no sign of any flaring. After that, the flare continues for about another $15-$ 18 frames. Finally, the flare dissipates and post-flare loops are observable (20 frames). The flare event transfers momentum to the surrounding medium, causing loops to oscillate. In addition, the whole region evacuates - the flare appears to blast material away, or at least change its temperature enough to put the 

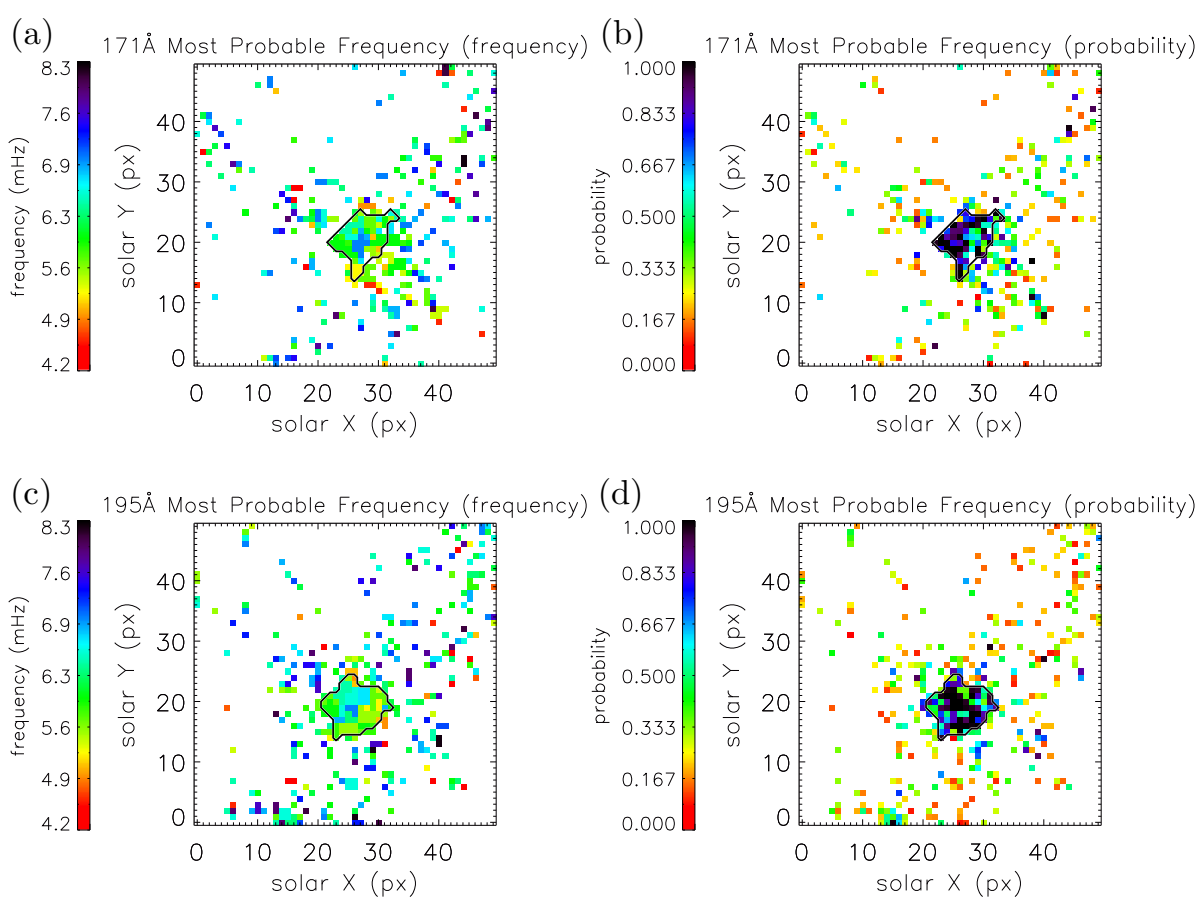

Figure 6. Zoomed in view of single large oscillation region found in Figures 4 and 5 Panels (a) and (b) are from the $171 \AA$ data, and panels (c) and (d) are derived from the $195 \AA$ data $(1$ July 1998).

material out of the TRACE $171 \AA$ passband. The physical phenomena captured by these observations imply that the resultant time-series have a significant background trend which varies from location to location. The detrending timescale (500 seconds) removes the secular background intensity variations such as flaring and dimming.

Results for the analysis in a three (120-240 seconds) and five minute frequency band (240-360 seconds) are given in Figure 9 and Figure 10 repestively, and Table 3. Only three three-minute frequency band regions are found. Two of them (regions 1 and 3) are distant from the central active region over relatively dark pieces of corona where the signal is weak. Region 2 (the largest of the three) overlies a portion of the active region where many oscillations are detected in the five-minute frequency band; the detection in the three-minute frequency band may be due to multiple loops each oscillating in the five-minute frequency band that coincidentally gives the appearance of a three-minute oscillation.

The detection algorithm finds eight of the nine transversely oscillating as described in Aschwanden et al. (1999) (their Figure 1, oscillations 1 through 9 excepting 5). Many of the other claimed detections of Figure 10] are in small regions distant from the flare site. These locations also typically show an oscillation which is more of a gentle sway, that is, the oscillation decays very quickly. It is instructive to consider Figure 3. (e) in comparison to Figure 3.(b). The spatial probability distribution is much denser at all lengthscales in Figure 3(e) than 
(a)

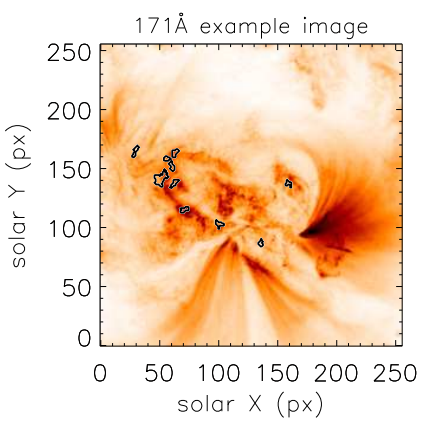

(c)

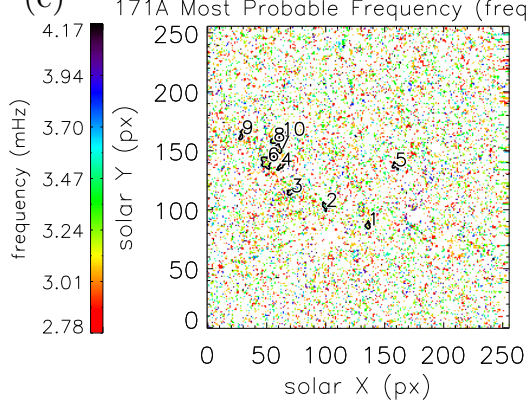

(e)

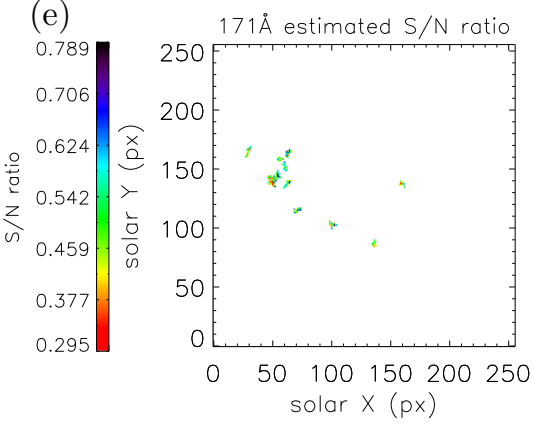

(b) 171А Most Probable Frequency (probability)

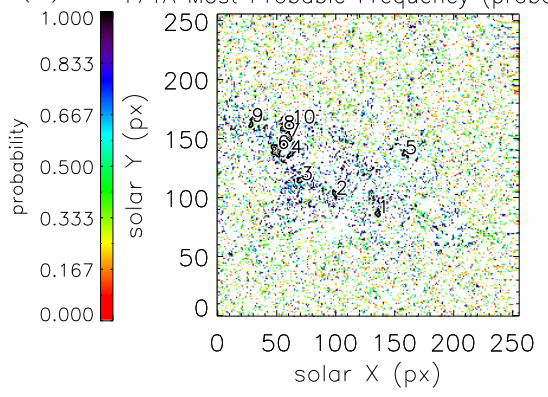

(d)

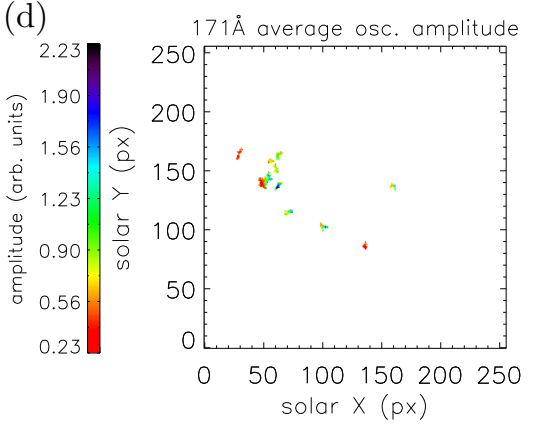

(f)

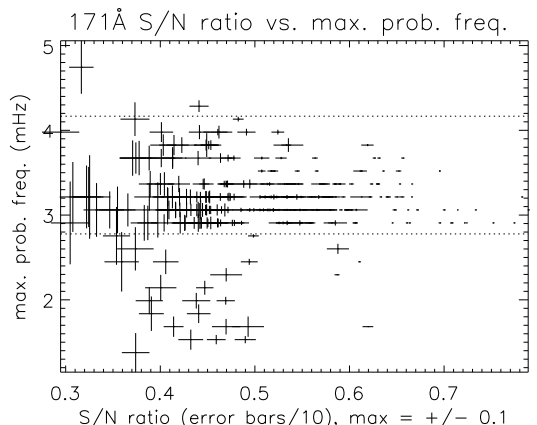

Figure 7. Example image data and results from the analysis algorithm for TRACE $171 \AA$ data taken on 1 July 1998 (panel (a)). Panel (b) is the probability that a given pixel supports an oscillation within the frequency band indicated in panel (c), in this case, a five-minute oscillation frequency band. Also indicated in panels $(\mathrm{a}-\mathrm{c})$ are the detected oscillation regions. Panels $(\mathrm{d}-\mathrm{f})$ show similar maps to those shown and described in Figure 4 Table 2 $\mathrm{c}$ ) shows the values for the detected region for the parameters listed in Table 1

in Figure 3(b). Figure 11 shows that the material of Figure 3(e) has a greater proportion of higher-probability oscillations than the material in Figure 3(b). Given the differing spatial distribution, it is clear that everywhere in TRACE field of view on 14 July 1998 was much more likely to oscillate in the five-minute frequency band. Since the probability map is filtered for regions that show a locally high probability, many more regions are found in this dataset than in the previous dataset. 
(a)

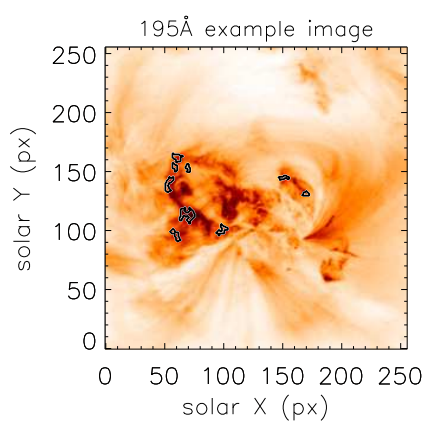

(c)

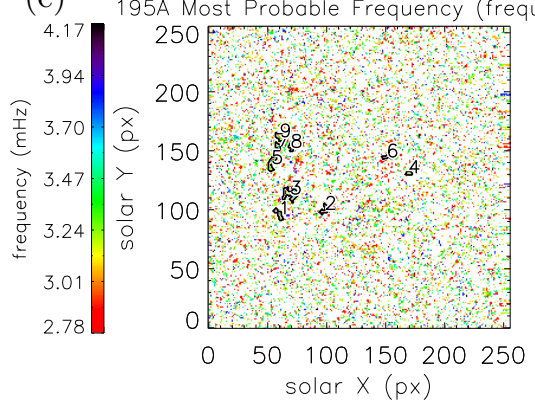

(e)

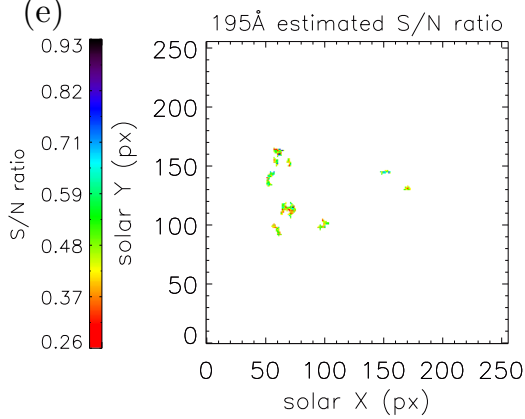

(b)

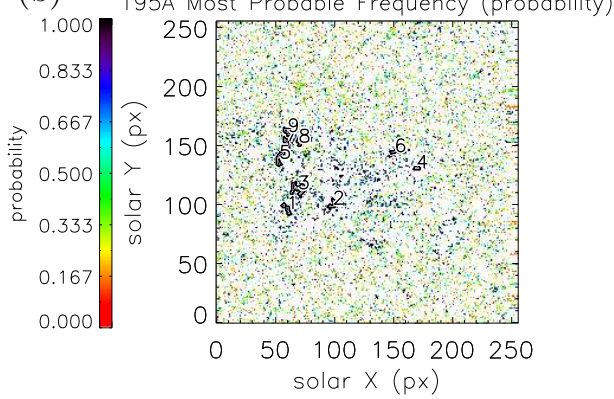

(d)

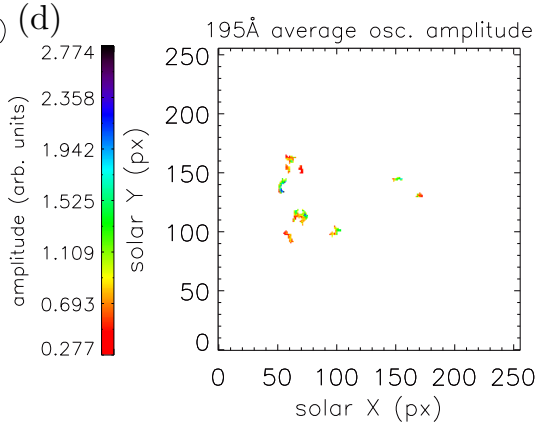

(f)

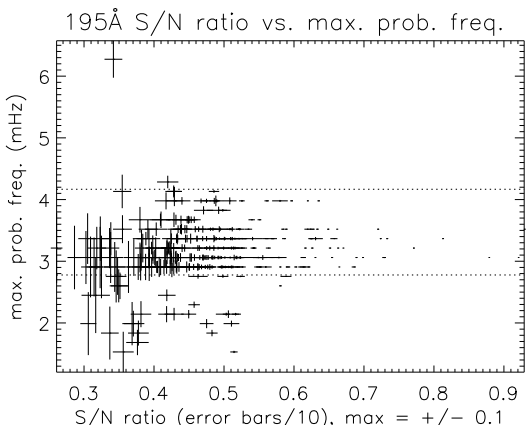

Figure 8. Example image data and results from the analysis algorithm for TRACE $195 \AA$ data taken on 1 July 1998 (panel (a)) in the five-minute frequency band. Panels (b-f) show similar maps to those shown and described in Figure 7 Table 21d) shows the values for the detected region for the parameters listed in Table 1

\section{Detecting Other Oscillatory Signals with this Algorithm}

This paper is primarily concerned with detecting areas in the solar atmosphere that oscillate with a single frequency, described by Equation (2). Other oscillations have been found in the solar atmosphere that are not perfectly described by Equation (2). Bretthorst (1988, ch. 6.1.4) notes that Equation (2) still gives strongly peaked probability distributions close to the true frequencies even when the actual signal exhibits features not present in the model, such as periodic but non-harmonic oscillations and non-stationary and non-Gaussian noise. In 
(a)

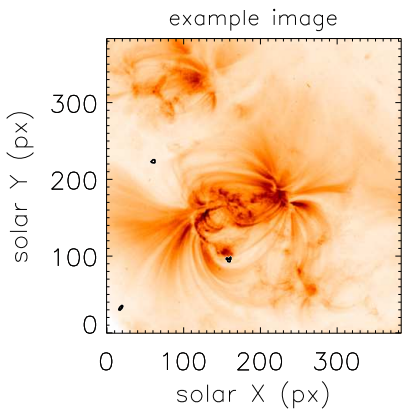

(c)

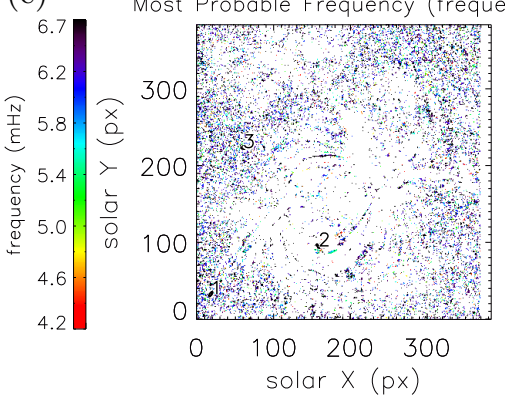

(e)

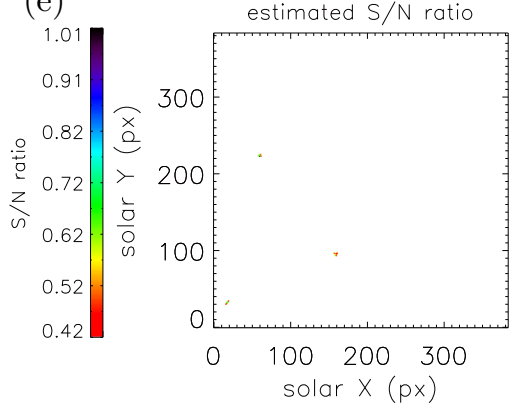

(b) Most Probable Frequency (probability)

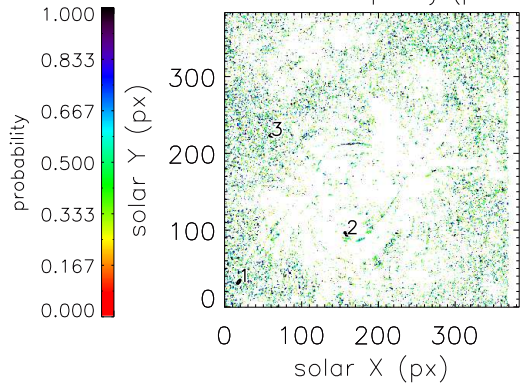

(d)

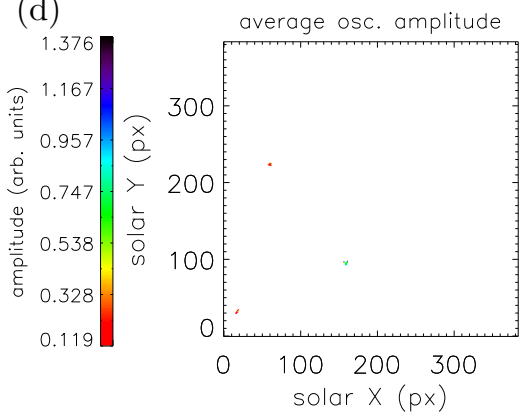

(f)

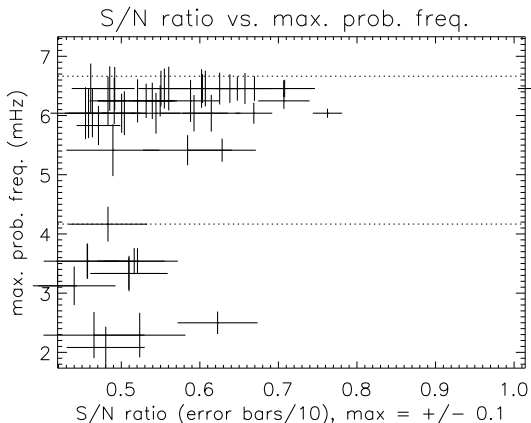

Figure 9. Example image data and results from the analysis algorithm for TRACE $171 \AA$ data taken on 14 July 1998 (panel (a)) in the three-minute frequency band. Panels (b-f) show similar maps to those shown and described in Figure 7 Panels $(b-f)$ show similar maps to those shown and described in 7 Table 3 shows the values for the detected region for the parameters listed in Table 1

the sections below we discuss some commonly occuring periodic signals in solar atmosphere, that contain features not modeled by Equation (2), and their effect on the probability distributions that are at the center of the proposed detection algorithm. 
(a)

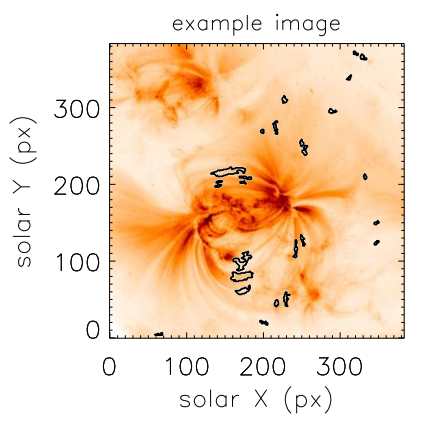

(c)

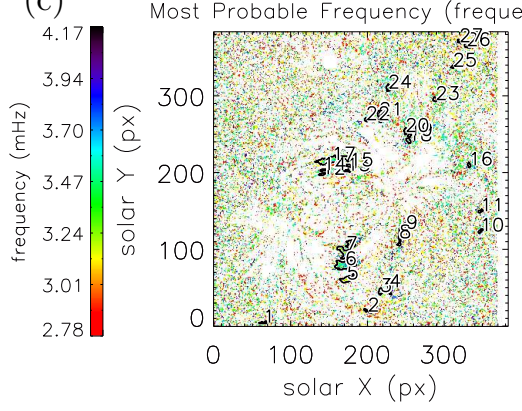

(e)

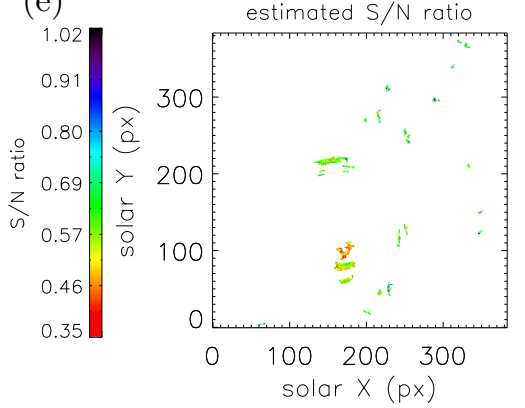

(b)

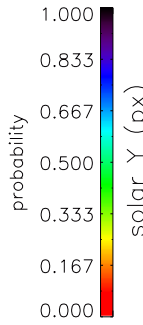

Most Probable Frequency (probability)

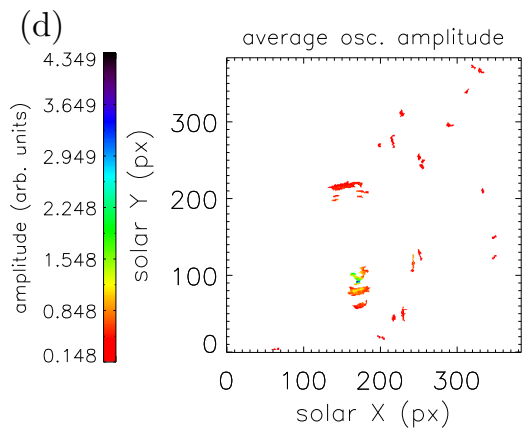

(f)

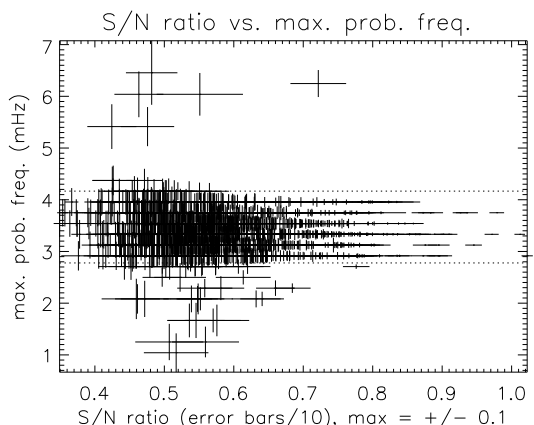

Figure 10. Example image data and results from the analysis algorithm for TRACE $171 \AA$ data taken on 14 July 1998 (panel (a)) in the five-minute frequency band. Panels (b-f) show similar maps to those shown and described in Figure 7 Panels $(\mathrm{b}-\mathrm{f}$ ) show similar maps to those shown and described in Figure 7 Table 3 shows the values for the detected region for the parameters listed in Table 1

\subsection{Decaying Oscillations}

Figure 12 applies the single-frequency model to an example dataset based on the transverse loop oscillation described by Nakariakov et al. (1999). The data (shown in Figure 12(a)) exhibits approximately the same properties as the true observation. Figure 12(b) shows the measured frequency to be close to the true frequency of $3.9 \mathrm{mHz}$. For comparison, Figure 12 (c) shows the same time-series as Figure 12(a) except with no decay, along with the probability distribution 
Table 3. Quantities reported (see Table 1) for the automatically detected regions found in 14 July 1998 TRACE $171 \AA$ - see Figure 10 for maps and plots of the detected regions. The quantities $Q$ and $E$ are also listed with their rank $r$ when compared to all other regions found in the same dataset. Region 4 is the same region as that studied by Nakariakov et al. (1999) and Ireland and De Moortel (2002), and region 8 is the base of the same loop. The numbers $A x$ in the region column refer to the oscillations found manually in Figure 1 of Aschwanden et al. (1999).

\begin{tabular}{|c|c|c|c|c|c|c|c|}
\hline region & $\bar{f} \pm \sigma_{f}$ & $A$ & $A_{0.95}$ & $F$ & $\bar{p}$ & $Q \cdot[r(Q)]$ & $E \cdot[r(E)]$ \\
\hline \multicolumn{8}{|l|}{$3 \min$} \\
\hline 1 & $6.303 \pm 0.455$ & 15 & 9 & 0.938 & 0.879 & $0.824[2]$ & $12.354[3]$ \\
\hline 2 & $6.383 \pm 0.264$ & 20 & 0 & 1.000 & 0.723 & $0.723[3]$ & $14.466[1]$ \\
\hline 3 & $6.547 \pm 0.151$ & 16 & 5 & 1.000 & 0.849 & $0.849[1]$ & $13.581[2]$ \\
\hline \multicolumn{6}{|l|}{$5 \mathrm{~min}$} & $\cdot$ & \\
\hline 1 & $3.319 \pm 0.052$ & 16 & 6 & 0.941 & 0.815 & $0.767[10]$ & $12.279[22]$ \\
\hline 2 & $3.076 \pm 0.127$ & 22 & 1 & 0.917 & 0.782 & $0.717[22]$ & $15.765[16]$ \\
\hline 3 & $3.408 \pm 0.348$ & 30 & 3 & 0.938 & 0.781 & $0.732[18]$ & $21.958[12]$ \\
\hline 4 & $3.811 \pm 0.185$ & 53 & 26 & 0.930 & 0.862 & $0.802[4]$ & $42.492[6]$ \\
\hline 5 & $3.117 \pm 0.164$ & 93 & 3 & 0.979 & 0.778 & $0.762[12]$ & $70.856[4]$ \\
\hline $6[A 3,8]$ & $3.616 \pm 0.290$ & 236 & 14 & 0.992 & 0.745 & $0.739[16]$ & $174.427[1]$ \\
\hline $7[A 7]$ & $3.477 \pm 0.358$ & 197 & 1 & 0.985 & 0.644 & $0.634[27]$ & $124.969[3]$ \\
\hline $8[A 4]$ & $3.556 \pm 0.239$ & 54 & 13 & 1.000 & 0.834 & $0.834[1]$ & $45.025[5]$ \\
\hline $9[A 9]$ & $3.038 \pm 0.116$ & 34 & 2 & 1.000 & 0.745 & $0.745[15]$ & $25.317[9]$ \\
\hline 10 & $3.415 \pm 0.323$ & 15 & 6 & 0.882 & 0.856 & $0.756[13]$ & $11.336[26]$ \\
\hline 11 & $3.163 \pm 0.231$ & 16 & 4 & 0.941 & 0.832 & $0.783[9]$ & $12.534[21]$ \\
\hline 12 & $3.482 \pm 0.275$ & 18 & 7 & 1.000 & 0.823 & $0.823[3]$ & $14.821[17]$ \\
\hline 13 & $3.410 \pm 0.213$ & 16 & 5 & 1.000 & 0.823 & $0.823[2]$ & $13.175[20]$ \\
\hline 14 & $3.306 \pm 0.262$ & 16 & 1 & 1.000 & 0.716 & $0.716[24]$ & 11.459 [25] \\
\hline 15 & $3.546 \pm 0.145$ & 36 & 5 & 0.947 & 0.757 & $0.718[20]$ & $25.835[8]$ \\
\hline 16 & $3.430 \pm 0.182$ & 17 & 1 & 0.944 & 0.693 & $0.654[26]$ & $11.126[27]$ \\
\hline $17[A 1,2,6]$ & $3.359 \pm 0.222$ & 247 & 35 & 0.939 & 0.749 & $0.703[25]$ & $173.700[2]$ \\
\hline 18 & $3.380 \pm 0.272$ & 26 & 8 & 0.963 & 0.764 & $0.736[17]$ & $19.134[15]$ \\
\hline 19 & $3.332 \pm 0.108$ & 16 & 3 & 0.941 & 0.761 & $0.716[23]$ & $11.463[24]$ \\
\hline 20 & $3.455 \pm 0.132$ & 27 & 7 & 1.000 & 0.788 & $0.788[7]$ & $21.274[14]$ \\
\hline 21 & $3.270 \pm 0.248$ & 44 & 7 & 0.936 & 0.773 & $0.724[19]$ & $31.839[7]$ \\
\hline 22 & $3.436 \pm 0.215$ & 16 & 3 & 0.941 & 0.762 & $0.717[21]$ & $11.475[23]$ \\
\hline 23 & $3.047 \pm 0.235$ & 30 & 8 & 0.968 & 0.819 & $0.792[6]$ & $23.767[11]$ \\
\hline 24 & $3.376 \pm 0.186$ & 33 & 12 & 0.917 & 0.833 & $0.764[11]$ & $25.203[10]$ \\
\hline 25 & $3.185 \pm 0.263$ & 17 & 4 & 1.000 & 0.787 & $0.787[8]$ & $13.377[19]$ \\
\hline 26 & $3.124 \pm 0.176$ & 29 & 8 & 0.935 & 0.799 & $0.748[14]$ & 21.684 [13] \\
\hline 27 & $3.173 \pm 0.202$ & 17 & 2 & 1.000 & 0.799 & $0.799[5]$ & $13.583[18]$ \\
\hline
\end{tabular}

for the frequency in Figure 12 (d). The only difference between the two is the error in the determination of the frequency. This is because the decay in the first time-series decreases the signal to noise ratio, and so later portions of the timeseries contribute less information to the determination of the frequency, and so the error increases. This shows that for decaying oscillations of the type already observed, the probability based methods of Section 2 yield good results. Further, 


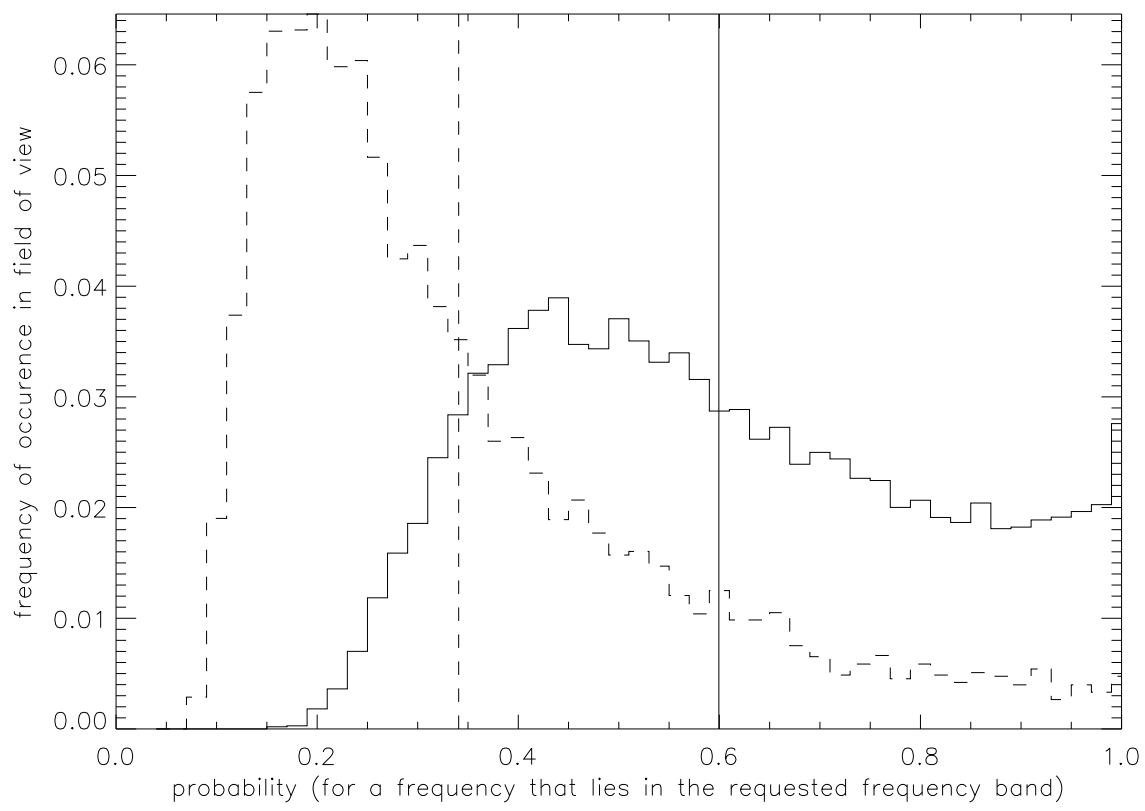

Figure 11. Distribution of non-zero probabilities for the 1 July and 14 July $171 \AA$ data. The dashed line refers to the 1 July $171 \AA$ data analyzed in the three-minute frequency band (120-240 seconds), as shown in Figure 3(b). The solid line refers to the 14 July $171 \AA$ data analyzed in the five-minute frequency band (240-360 seconds), as shown in Figure 3) (e). Average values to each frequency distribution are indicated by the vertical lines of the same line style. See Section 4.2 for more detail.

it also demonstrates that even although we used an inappropriate model, the model of Section 2 still gives a sufficiently peaked distribution at the right frequency to enable detection.

\subsection{Non-Stationary Frequency Oscillations}

Figure 13 applies the single-frequency model to an example dataset based on the "tadpole" signature of Nakariakov et al. (2004). The example data-set reproduces the extent of the signal as mentioned in Nakariakov et al. (2004), who claim that there is a time-varying (i.e., non-stationary) frequency present in a signal found by Katsiyannis et al. (2003) (see also Williams et al., 2001, Williams et al. 2002) in the Solar Eclipse Imaging System (SECIS) of Queens University, Northern Ireland eclispe data taken on 11 August 1999. Their observation is modeled as having a signal to noise ratio of 1.5, and a non-stationary frequency that lowers by $5 \%$ over the range (Figure $13(\mathrm{a})$ ). The result of an analysis using Equation (12) with Equation (2) (note that Equation (3), as used in the automated detection algorithm, is essentially the same) is shown in Figure 13(b). Figure 13(c) shows the same extent of signal except that now the oscillation is stationary. Note that the algorithm will not detect the oscillation as being non-stationary or as not extending across the observation range, as neither 
(a) time series - decay

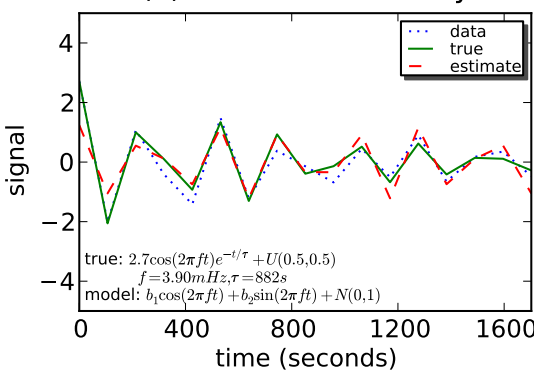

(c) time series - no decay

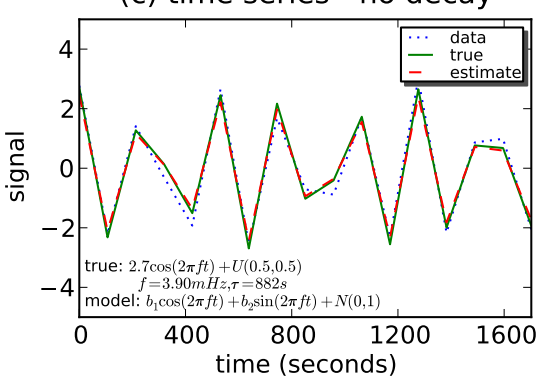

(b) probability

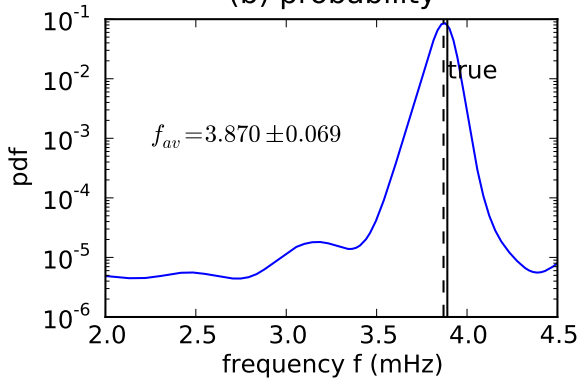

(d) probability

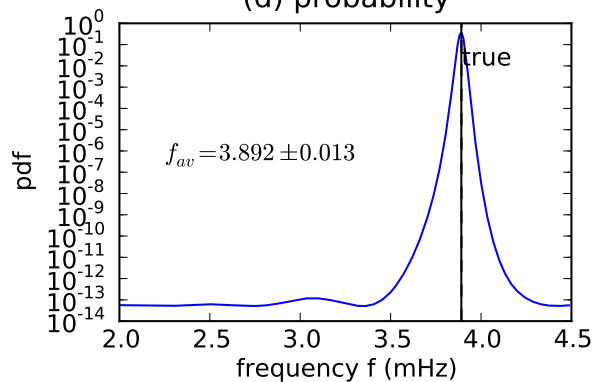

Figure 12. Effect of an exponential decay in the observed signal on the detection of an oscillation. Panel (a) shows the observed noisy time series signal (dotted line), the true (non-noisy) signal (solid line) and the estimated signal (dashed line). Panel (b) shows the probability-density function for the observed signal as defined by Equation (3). Panels (c) and (d) are the same as (a) except the exponential decay is no longer present. The probability-density function in Panel (d) is much narrower than in Panel (b). The effect of the exponential decay is to make the location of the average frequency more uncertain. This is because the signal-to-noise ratio decreases due to the exponential decay and therefore there is less information to determine the frequency when compared to the observed signal of panel (c). See Section 5.1 for more detail.

of these effects are included in the model oscillation Equation (2). However, the probability distribution functions Figures $13(\mathrm{~b}, \mathrm{~d})$ are strongly and singly peaked, and they would therefore be detected given a wide enough detection window $\omega_{1}, \omega_{2}$ (see Equation 7 ).

\subsection{Multiple discrete frequencies}

The results stated in this paper are derived with regard to a single frequency model, Equation (2). Initial identification of a pixel as containing a significant oscillation relies on the integrated probability over a user-defined frequency band exceeding a high user-defined limit (in this case, 0.95, see Section 3.3). If there are multiple frequencies inside the user-defined frequency band and the integrated probability exceeds 0.95 , then the algorithm will report a detection at that pixel and report the average frequency (and error) within that frequency band. It cannot do any more, since the model assumes the presence of only a single frequency. 
(a) nonstationary frequency, t>14

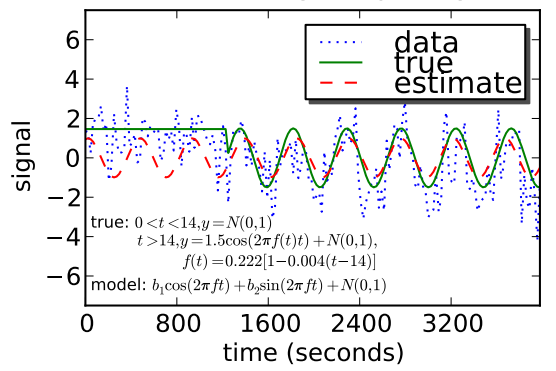

(c) stationary frequency, $t>14$

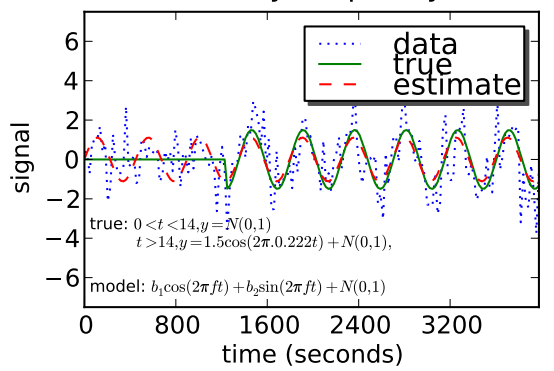

(b) probability

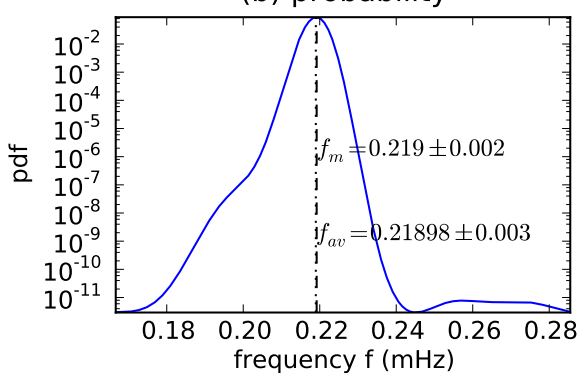

(d) probability

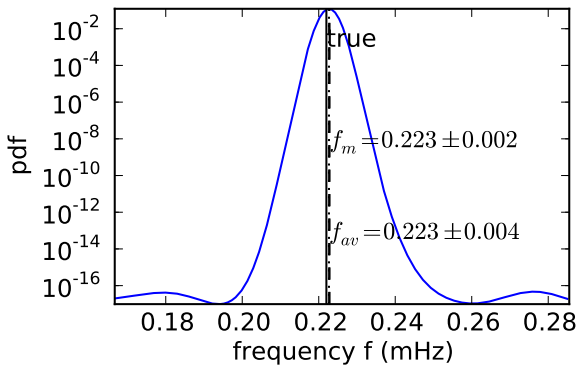

Figure 13. Effect of a non-stationary frequency in the observed signal on the detection of an oscillation. Panel (a) shows the observed noisy time series signal (dotted line), the true (non-noisy) signal (solid line) and the estimated signal (dashed line). The true signal consists of noise at $0<t<14$ and a time-varying frequency at $t>14$. Panel (b) shows the probability-density function for the observed signal as defined by Equation (3). Panels (c) and (d) show the same quantities as (a) and (b) respectively except that the frequency in the observed and true signals are constant at $t>14$. The peak of the probability-density in panel (b) is at a lower frequency than in panel (d) because the observed signal contains more low frequencies. Note that in both cases the probability-density function is strongly peaked and so most of the probability lies in a narrow band around the peak. See Section 5.2 for more detail.

Consider now the case of two well-separated frequencies such that one frequency lies inside the user-defined frequency band $\left(\omega_{1}, \omega_{2}\right.$, Equation (7)) and the other lies outside the user-defined frequency band (Figure [14 a). Both frequencies are present in the observation, and both have the same amplitude and signal-tonoise ratio. The model however, supposes the presence of one frequency. Hence the probability will be split between these two frequencies (Figure 14(b)). Which frequency has the highest probably depends on the evidence for each in the timeseries, that is, the number of oscillations in the time series and the signal-to-noise ratio for each frequency present. In the case above, the most probable frequency is in the three-minute wave band. If one were looking in this frequency band, then a peak would be detected and the algorithm would decide if there were enough probability in the user-defined frequency band $\left[\omega_{1}, \omega_{2}\right]$ to claim a detection. However if the user-defined frequency band $\left[\omega_{1}, \omega_{2}\right]$ was in the five-minute frequency band, then since the majority of the probability lies outside this range, no detection would be claimed. However, we note that observations of multiple 
(a) time series - decay

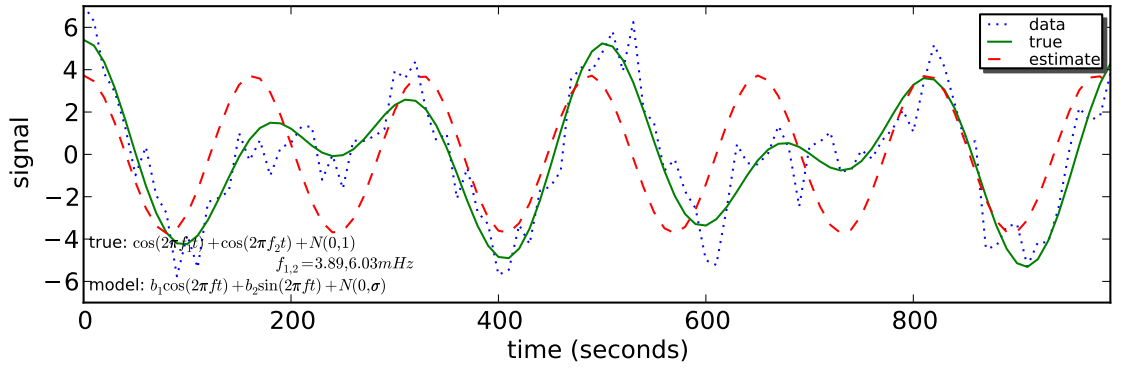

(b) probability

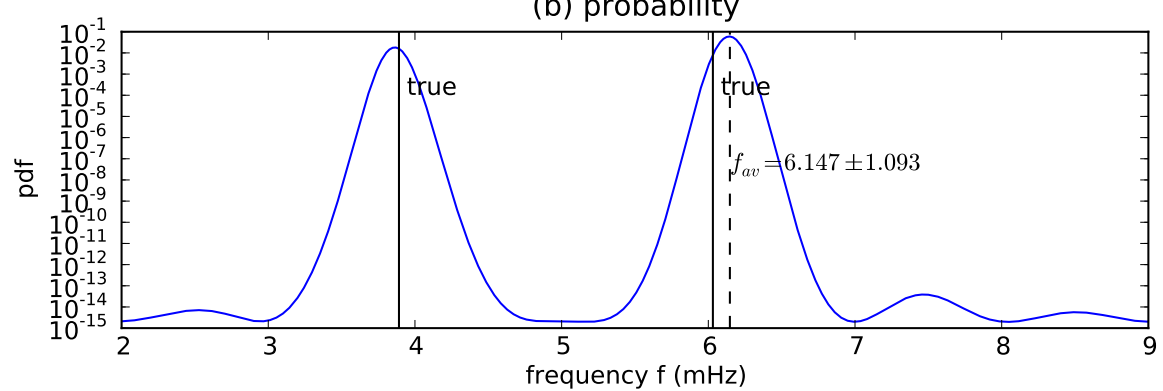

Figure 14. Analysis of a time-series that has two widely separated present. In panel (a), the observed signal (dotted line) is a noisy observation of the true signal (solid). The estimated signal (dashed) is found using Equation (3), a single frequency model. Panel (b) shows the probability-density function (Equation (3) of the observed signal. In this case, the maximum probability density is close to the frequency of the higher frequency signal because the signal contains more information about this frequency than the lower frequency. See Section 5.3 for more detail.

oscillations in single structures in the corona show one dominant oscillation and a second, much weaker oscillation (Verwichte et al. (2004)). Therefore, the situation of Figure 14(a) - two oscillations of equal amplitude but different frequency - is observationally unlikely in the corona. However, one may use the approach here to first identify pixels that have a dominant oscillation present, and use other methods to examine for the presence of secondary, smaller amplitude oscillations.

Deciding how many frequencies a time-series supports is specifically excluded by the model choice at the start, that is, a single oscillation plus noise. To properly decide how many frequencies are present in a time-series, one must define a model for each case to be considered (either no frequencies, one frequency, two frequencies, three frequencies, etcetera), calculate the probability of each model, and decide which model is the most probable. Bayesian probability tests hypotheses (given the data), and those hypotheses must be explicitly stated. Introducing other hypotheses, such as multiple frequency models, is asking a different question of the data. For example, multiple frequencies have long been observed in the type of sunspot observations examined by Marsh, Ireland, and Kucera (2008), and therefore a single-oscillation model is inappropriate to start with; one simply would not use this model in this case. In addition, the approach taken by 
Marsh, Ireland, and Kucera (2008) is prohibitively expensive computationally at present, and so is not suitable for the automated detection of oscillations in large datasets.

\section{Conclusion}

Oscillations in the solar atmosphere have already demonstrated their worth as probes of the physical conditions present. SDO will be a major contributor to the study of solar atmospheric oscillations, and automated detection algorithms will be necessary in order to maximize the scientific potential here. The algorithm parameters in the filtering procedure (see Section [3.3) will have to be tailored to SDO data; other algorithms will also probably have to undergo a "tweaking" phase to operate optimally.

The algorithm described here is a first attempt at implementing an automated coronal oscillation detector based on a Bayesian understanding of probability. It shows promise in being able to find areas of the solar atmosphere that are highly likely to support an oscillation. Section 4 shows that it is able to find both longitudinal and transversely oscillating loops at low estimated signal-to-noise ratio over a complex background scene (the non-oscillating or slowly varying background, where slowly varying is understood as evolution longer than the characteristic timescale of the background trend subtraction). We note that even by eye, these events appear to be at low signal-to-noise ratio, and the estimates calculated here (Figures 4-10) agree with this observation.

We also examined the same quiet-Sun TRACE 1600 and $1700 \AA$ data as McIntosh, Fleck, and Tarbell (2004). In that paper, the authors calculate the travel time of waves in two different UV wave bands, with the understanding that the entire FOV contains oscillations. We find that almost the entire FOV contains oscillations. The algorithm returns positive detections over almost all of the FOV for other TRACE UV data (McAteer et al., 2004). In both cases the detection itself takes only a few seconds: however, the other parameters such as signal-to-noise ratio takes many multiples of the detection time. This suggests that a line production version which generates data products similar to Figures 4 10, or 6 be restricted to data where we know that only a small percentage of the FOV contains a signal, such as the higher temperature corona. An alternate mode of operation is that the reported quantities are restricted to detections only, with further quantities (such as signal to noise ratios) calculated offline by the interested user.

The model oscillation that Equation (2) describes, for a single pixel, a sinusoidol intensity oscillation existing for the entire duration of the time-series, in the presence of Gaussian noise. Although this seems quite a restrictive model, it is clear from the results of Section 4.2 that it is sufficient to enable the detection of decaying transverse oscillations. Indeed, Ireland and De Moortel (2002) showed that the oscillation studied by Nakariakov et al. (1999) (region 4 in Table 3) displays non-Gaussian noise and a linear change in period over the measurable duration of the oscillatory motion, complications not contained in the model choice, Equation (2). A transverse oscillation on a single pixel appears as a periodic non-sinusoidal change in intensity as the loop swipes across the field of view. 
Despite the great difference between the model choice and the appearance of such an oscillation in this analysis, the resemblance between the two is sufficient for our algorithm to be able to detect transversely oscillating material. This is an example of the sufficiency of the model, (Equation (2)), to adequately describe a wide range of periodic behavior, as previously noted by Bretthorst (1988).

The algorithm runs quickly on the data (less than a minute, reported in Table 4) on an Apple MacBook (dual core, 1.6 Ghz processor), without any special efforts at algorithmic optimization. Analysis of binned (prepared) full-disk SDO data (see Table 4) is possible with existing computers. Note, however, that the analysis times quoted do not take into account the time required to prepare the data for automated oscillation analysis. This overhead will presumably be the same for all detection algorithms, and must be considered in design of a full-scale operational SDO oscillation-detection algorithm in order for it to run faster than the time taken to acquire the data.

This paper introduces two new features to the discussion of automated detection of oscillations in the solar atmosphere. Firstly, the algorithm is implemented using a Bayesian interpretation of probability as a "degree of belief" as opposed to the standard interpretation as "frequency of occurence" (leading to powerful and convenient formulae such as Equations 12 and 3 . At the core of the algorithm lies the probability that solar atmospheric time-series can be described as a single sinusoidal oscillation at a fixed frequency, subject to distortion Gaussian noise. As Bretthorst (1988) notes, this is a good approximation for many purposes. This algorithm does not say anything about the presence of two or more sinusoidal signals in a single time-series. However, it is certainly possible to develop an analysis algorithm to assign probabilities to each of the three hypotheses that the time time-series is either noise, contains a single frequency or contains two frequencies. Most of the required mathematics is quoted or referred to in this work; such an algorithm will be the described in future developments.

Secondly, we have introduced "quality measures" in an attempt to grade the regions that survive the region finding and filtering process. This appears to be necessary given the large number of oscillating regions that can occur in a given dataset, for example the 14 July 1998 TRACE $171 \AA$ data. Further criteria may be set by individual users. For example high values of the ratio $A_{0.95} / A$ indicate that the region has a high proportion of pixels very probably supporting a frequency; a threshold could be set to filter only those regions that have a high proportion of very certain pixels. In addition, the current VOEvent standard makes for the provision of extra algorithm information such as arbitrary parameters $(R, m)$ to be carried along with any results. This means the user will be fully informed of all the parameter values used to obtain the results.

Future algorithm development will concentrate on improving the probability map filtering (step 6 of Figure 2, and also Section 3.3), extending the analysis to assign probabilities to multi-frequency models, and to distinguishing between longitudinal and transverse oscillations (it may be possible to assign a probability that a given wave-mode has been observed). Understanding the wave mode demands an understanding of the structure on which it is supported, which naturally leads to the automated detection and characterization of loop structures, which is a complex topic by itself (see Aschwanden et al. (2008) for 
Table 4. Performance of the algorithm on the analyzed data compared to data acquisition time. Also shown is the projected performance on SDO data.

\begin{tabular}{ccc} 
data & data size & algorithm time [observation duration] \\
\hline 1 July & $256 \times 256$ & $30[6231]$ \\
& 201 samples & \\
& 31 s cadence & \\
& $368 \times 368$ & $25[3431]$ \\
14 July & 47 samples & \\
& 31 s cadence & \\
& $4096 \times 4096$ & \\
raw SDO & 200 samples & \\
& 10 s cadence & $92000]$ \\
& $2048 \times 2048$ & \\
$2 \times 2 \times 2$ rebinned & 200 samples \\
SDO & 20 s cadence
\end{tabular}

a review). We hope that the algorithm presented here is a first step towards automated coronal seismology.

Acknowledgements This work was supported by the NASA SESDA contract and a NASA Heliophysics Guest Investigator award (NNG08EL33C). SOHO is a joint project of international co-operation by ESA and NASA.

\section{References}

Aschwanden, M.J., Fletcher, L., Schrijver, C.J., Alexander, D.: 1999, Coronal Loop Oscillations Observed with the Transition Region and Coronal Explorer. Astrophys. J. 520, 880-894. doi $10.1086 / 307502$

Aschwanden, M.J., de Pontieu, B., Schrijver, C.J., Title, A.M.: 2002, Transverse Oscillations in Coronal Loops Observed with TRACE II. Measurements of Geometric and Physical Parameters. Solar Phys. 206, 99-132. doi 10.1023/A:1014916701283

Aschwanden, M.J., Lee, J.K., Gary, G.A., Smith, M., Inhester, B.: 2008, Comparison of Five Numerical Codes for Automated Tracing of Coronal Loops. Solar Phys. 248, 359-377. doi $10.1007 /$ s11207-007-9064-9.

Bayes, T.: 1763, An Essay towards solving a Problem in the Doctrine of Chances. Phil. Trans. Roy. Soc. 53, 370-418. http://rstl.royalsocietypublishing.org/content/53/370.

Berger, T.E., de Pontieu, B., Fletcher, L., Schrijver, C.J., Tarbell, T.D., Title, A.M.: 1999, What is Moss? Solar Phys. 190, 409-418. doi 10.1023/A:1005286503963

Berghmans, D., Clette, F.: 1999, Active region EUV transient brightenings - First Results by EIT of SOHO JOP80. Solar Phys. 186, 207-229.

Bretthorst, G.L.: 1988, Lecture Notes in Statistics: Bayesian Spectrum Analysis and Parameter Estimation, Springer, Berlin Heidelberg.

Chatfield, C.: 1996, The analysis of time series: an introduction, 5th edn. Chapman and Hall, Florida.

Cooley, J., Tukey, J.: 1965, An Algorithm for the Machine Computation of Complex Fourier Series . Math. Comp. 19, 297-301.

De Moortel, I.: 2005, An overview of coronal seismology. Roy. Soc. London Phil. Trans. Series A 363, 2743-2760.

De Moortel, I., Hood, A.W.: 2003, The damping of slow MHD waves in solar coronal magnetic fields. Astron. Astrophys. 408, 755-765. doi 10.1051/0004-6361:20030984 
De Moortel, I., Hood, A.W.: 2004, The damping of slow MHD waves in solar coronal magnetic fields. II. The effect of gravitational stratification and field line divergence. Astron. Astrophys. 415, 705-715. doi 10.1051/0004-6361:20034233.

De Moortel, I., McAteer, R.T.J.: 2004, Waves and wavelets: An automated detection technique for solar oscillations. Solar Phys. 223, 1-11. doi 10.1007/s11207-004-0806-7

De Moortel, I., Ireland, J., Walsh, R.W.: 2000, Observation of oscillations in coronal loops. Astron. Astrophys. 355, L23-L26.

De Moortel, I., Ireland, J., Hood, A.W., Walsh, R.W.: 2002, The detection of 3 and 5 min period oscillations in coronal loops. Astron. Astrophys. 387, L13-L16. doi 10.1051/0004-6361:20020436

de Pontieu, B., Erdélyi, R., De Moortel, I.: 2005, How to Channel Photospheric Oscillations into the Corona. Astrophys. J. Lett. 624, L61-L64. doi 10.1086/430345

de Pontieu, B., Erdélyi, R., James, S.P.: 2004, Solar chromospheric spicules from the leakage of photospheric oscillations and flows. Nature 430, 536-539. doi 10.1038 /nature02749.

de Pontieu, B., Berger, T.E., Schrijver, C.J., Title, A.M.: 1999, Dynamics of Transition Region 'Moss' at high time resolution. Solar Phys. 190, 419-435. doi 10.1023/A:1005220606223.

Deforest, C.E., Gurman, J.B.: 1998, Observation of Quasi-periodic Compressive Waves in Solar Polar Plumes. Astrophys. J. Lett. 501, L217. doi 10.1086/311460

Fletcher, L., de Pontieu, B.: 1999, Plasma Diagnostics of Transition Region "Moss" using SOHO/CDS and TRACE. Astrophys. J. Lett. 520, L135-L138. doi 10.1086/312157

Grechnev, V.V.: 2003, A method to analyze imaging radio data on solar flares. Solar Phys. 213, $103-110$.

Gregory, P.C.: 2005, Bayesian Logical Data Analysis for the Physical Sciences: A Comparative Approach with 'Mathematica' Support, Cambridge University Press, Cambridge, UK.

Ireland, J., De Moortel, I.: 2002, Application of wavelet analysis to transversal coronal loop oscillations. Astron. Astrophys. 391, 339-351. doi 10.1051/0004-6361:20020643.

Ireland, J., Walsh, R.W., Harrison, R.A., Priest, E.R.: 1999, A wavelet analysis of active region oscillations. Astron. Astrophys. 347, 355-365.

Jaynes, E.T.: 1987, Bayesian spectrum and chirp analysis. In: Smith, C.R., Erickson, G.J. (eds.) Maximum Entropy and Bayesian Spectral Analysis and Estimation Problems, $1-37$.

Jaynes, E.T.: 2003, Probability Theory: The Logic Of Science, 1st edn. Cambridge University Press, Cambridge, United Kingdom.

Katsiyannis, A.C., Williams, D.R., McAteer, R.T.J., Gallagher, P.T., Keenan, F.P., Murtagh, F.: 2003, Eclipse observations of high-frequency oscillations in active region coronal loops. Astron. Astrophys. 406, $709-714$. doi 10.1051/0004-6361:20030458

King, D.B., Nakariakov, V.M., Deluca, E.E., Golub, L., McClements, K.G.: 2003, Propagating EUV disturbances in the Solar corona: Two-wavelength observations. Astron. Astrophys. 404, L1 - L4. doi 10.1051/0004-6361:20030763

Linnell-Nemec, A.F., Nemec, J.M.: 1985, A test of significance for periods derived using phasedispersion-minimization techniques. Astronom. J. 90, 2317-2320. doi $10.1086 / 113936$

Marsh, M.S., Ireland, J., Kucera, T.: 2008, Bayesian Analysis of Solar Oscillations. Astrophys. J. 681, 672-679. doi $10.1086 / 588751$

McAteer, R.T.J., Gallagher, P.T., Bloomfield, D.S., Williams, D.R., Mathioudakis, M., Keenan, F.P.: 2004, Ultraviolet Oscillations in the Chromosphere of the Quiet Sun. Astrophys. J. 602, 436-445. doi $10.1086 / 380835$.

McIntosh, S.W., de Pontieu, B., Tomczyk, S.: 2008, A Coherence-Based Approach for Tracking Waves in the Solar Corona. Solar Phys. 252, 321-348. doi 10.1007/s11207-008-9257-x

McIntosh, S.W., Fleck, B., Tarbell, T.D.: 2004, Chromospheric Oscillations in an Equatorial Coronal Hole. Astrophys. J. Lett. 609, L95-L98. doi 10.1086/422748

Nakariakov, V.M., King, D.B.: 2007, Coronal Periodmaps. Solar Phys. 241, 397-409. doi $10.1007 /$ s11207-007-0348-x

Nakariakov, V.M., Verwichte, E.: 2005, Coronal Waves and Oscillations. Living Reviews in Solar Physics 2, 3 .

Nakariakov, V.M., Ofman, L., Deluca, E.E., Roberts, B., Davila, J.M.: 1999, TRACE observation of damped coronal loop oscillations: Implications for coronal heating. Science 285, 862 - 864. doi $10.1126 /$ science.285.5429.862

Nakariakov, V.M., Arber, T.D., Ault, C.E., Katsiyannis, A.C., Williams, D.R., Keenan, F.P.: 2004, Time signatures of impulsively generated coronal fast wave trains. Mon. Not. Roy. Astron. Soc. 349, 705-709. doi 10.1111/j.1365-2966.2004.07537.x 
Nightingale, R.W., Aschwanden, M.J., Hurlburt, N.E.: 1999, Time Variability of EUV Brightenings in Coronal Loops Observed with TRACE. Solar Phys. 190, 249-265. doi $10.1023 / \mathrm{A}: 1005211618498$.

Ofman, L., Romoli, M., Poletto, G., Noci, G., Kohl, J.L.: 1997, Ultraviolet Coronagraph Spectrometer Observations of Density Fluctuations in the Solar Wind. Astrophys. J. Lett. 491, L111. doi $10.1086 / 311067$

Ó Ruanaidh, J.K., Fitzgerald, W.J.: 1996, Numerical Bayesian methods applied to signal processing, Springer, New York/London.

O'Shea, E., Banerjee, D., Doyle, J.G., Fleck, B., Murtagh, F.: 2001, Active region oscillations. Astron. Astrophys. 368, 1095-1107. doi:10.1051/0004-6361:20010073

Robbrecht, E., Verwichte, E., Berghmans, D., Hochedez, J.F., Poedts, S., Nakariakov, V.M.: 2001, Slow magnetoacoustic waves in coronal loops: EIT and TRACE. Astron. Astrophys. 370, 591 - 601. doi:10.1051/0004-6361:20010226

Schuster, A.: 1898, On the Investigation of Hidden Periodicities with Application to a Supposed 26 Day Period of Meteorological Phenomena. Terr. Mag. 3, 13-13.

Sych, R.A., Nakariakov, V.M.: 2008, The Pixelised Wavelet Filtering Method to Study Waves and Oscillations in Time Sequences of Solar Atmospheric Images. Solar Phys. 248, $395-$ 408. doi $10.1007 / \mathrm{s} 11207-007-9005-7$

Torrence, C., Compo, G.P.: 1998, A Practical Guide to Wavelet Analysis. Bull. Am. Meteor. Soc. 79, $61-78$. doi $10.1175 / 1520-0477(1998) 079$

Verwichte, E., Nakariakov, V.M., Ofman, L., Deluca, E.E.: 2004, Characteristics of transverse oscillations in a coronal loop arcade. Solar Phys. 223, $77-94$. doi $10.1007 /$ s11207-004-0807-6

Williams, D.R., Phillips, K.J.H., Rudawy, P., Mathioudakis, M., Gallagher, P.T., O'Shea, E., Keenan, F.P., Read, P., Rompolt, B.: 2001, High-frequency oscillations in a solar active region coronal loop. Mon. Not. Roy. Astron. Soc. 326, 428-436. doi $10.1046 /$ j.1365-8711.2001.04491.x

Williams, D.R., Mathioudakis, M., Gallagher, P.T., Phillips, K.J.H., McAteer, R.T.J., Keenan, F.P., Rudawy, P., Katsiyannis, A.C.: 2002, An observational study of a magnetoacoustic wave in the solar corona. Mon. Not. Roy. Astron. Soc. 336, 747-752. doi $10.1046 /$ j.1365-8711.2002.05764.x

\section{Appendix}

\section{A. The General Linear Model}

A time-series is a special case of a more general linear model description. In this section we briefly recap the argument of Ó Ruanaidh and Fitzgerald (1996) in deriving Equation (3). In this description, a signal $d(i)$ observed at times $t_{i}$ $(1 \leq i \leq N)$, is modeled as

$$
d(i)=\sum_{k=1}^{M} b_{k} g_{k}(i)+x(i)
$$

with $M$ basis functions $g_{k}(i)$, each of amplitude $b_{k}$, evaluated at time $t_{i}$ (parameterized by $\{\omega\}$ ) and Gaussian distributed noise $x(i)$ of mean 0 and standard deviation $\sigma$. In the context of Section 2 the observation $D$ is equivalent to the signal $d(i)$, and the hypothesis $H$ is that the data can be described by the right hand side of Equation (8), including the noise. In matrix form, the above equation may be written as

$$
\mathbf{d}=\mathbf{G b}+\mathbf{x}
$$


where $\mathbf{d}$ is a $N \times 1$ matrix of the observed data, and $\mathbf{x}$ is a $N \times 1$ matrix of identically distributed and independent Gaussian noise samples. The matrix $\mathbf{G}$ is size $N \times M$; each column of $\mathbf{G}$ is one of the basis functions evaluated at all $t_{i}$. The matrix $\mathbf{b}$ is a $M \times 1$ matrix, the linear coefficients of each of the (column) basis functions in $\mathbf{G}$. The likelihood function of the observed data is

$$
\begin{aligned}
p(\mathbf{d} \mid\{\omega\}, \sigma, \mathbf{b}, I) & =\frac{1}{\left(2 \pi \sigma^{2}\right)^{N / 2}} \exp \left[-\frac{\mathbf{x}^{T} \mathbf{x}}{2 \sigma^{2}}\right] \\
& =\frac{1}{\left(2 \pi \sigma^{2}\right)^{N / 2}} \exp \left[-\frac{(\mathbf{d}-\mathbf{G} \mathbf{b})^{T}(\mathbf{d}-\mathbf{G b})}{2 \sigma^{2}}\right]
\end{aligned}
$$

where $\{\omega\}$ parameterize the basis functions $g_{k}$ and hence $\mathbf{G}$. This equation is derived by multiplying together the probability distributions of the noise $x_{i}$ at each time $i$. The exponent shows that maximizing the probability is equivalent to minimizing the difference between the data and the basis functions (weighted by their amplitudes); indeed, this equation forms the basis of least-squares fitting in the presence of Gaussian noise.

In analysis, one is primarily interested in the values of the parameters $\{\omega\}$, and secondarily interested in the other values such as $\mathbf{b}$ and $\sigma$. Ó Ruanaidh and Fitzgerald (1996) and Bretthorst (1988) describe the process by which the "nuisance parameters" $\mathbf{b}$ and $\sigma$ are removed from further consideration by marginalization. The statement of Bayes' theorem for the general linear model, using Equations (11) and (10) is

$$
p(\{\omega\}, \mathbf{b}, \sigma \mid \mathbf{d}, I)=\frac{p(\{\omega\}, \mathbf{b}, \sigma \mid I) p(\mathbf{d} \mid\{\omega\}, \sigma, \mathbf{b}, I)}{p(\mathbf{d} \mid I)} .
$$

Integrating over $\mathbf{b}$ and $\sigma$ using a prior $p(\{\omega\}, \mathbf{b}, \sigma \mid I)$, removes these variables from further explicit consideration, and is an example of Bayesian marginalization. On integration, this obtains the marginal posterior distribution for the parameters $\{\omega\}$ :

$$
p(\{\omega\} \mid \mathbf{d}, I)=\int_{\mathbf{b}} \int_{\sigma} p(\{\omega\}, \mathbf{b}, \sigma \mid \mathbf{d}, I) \mathrm{d} \sigma \mathrm{d} \mathbf{b} .
$$

Ó Ruanaidh and Fitzgerald (1996) and Bretthorst (1988) use uniform priors for the amplitude parameters $\mathbf{b}(p(\mathbf{b}, I)=$ constant $)$ and the Jeffreys prior $(p(\sigma \mid I) \propto$ $1 / \sigma)$ for $\sigma$. On integration,

$$
p(\{\omega\} \mid, \mathbf{d}, I) \propto \frac{\left[\mathbf{d}^{T} \mathbf{d}-\mathbf{d}^{T} \mathbf{G}\left(\mathbf{G}^{T} \mathbf{G}\right)^{-1} \mathbf{G}^{T} \mathbf{d}\right]^{(M-N) / 2}}{\sqrt{\operatorname{det}\left(\mathbf{G}^{T} \mathbf{G}\right)}}
$$

Equation (12) is a function of $\{\omega\}$ only; the standard deviation (i.e., the noise level of the time-series) or the amplitude of the basis functions (the values $\mathbf{b}$ ) need not be known in order for estimates of $\{\omega\}$ to be found. This is a very powerful equation, with clear application to solar time-series analysis, where estimates of the noise level and oscillation amplitude in the data are often difficult to obtain 
by direct fitting of model functions to the observed time-series. It should also be noted that Equation (12) arises from Equations (9) and (10), which is the basis of a general least squares fit to the data given Gaussian distributed noise.

\section{B. Estimating Basis Function Amplitudes and Variance}

Maximizing Equation (10) with respect to $\mathbf{b}$ (on substitution of Equation (9)) leads to an amplitude estimate:

$$
\hat{\mathbf{b}}=\left(\mathbf{G}^{T} \mathbf{G}\right)^{-1} \mathbf{G}^{T} \mathbf{d} .
$$

This maximization is identical to the "least squares" fit to the data for a given value of $\{\omega\}$. Given these amplitudes, the model fit is then

$$
\hat{\mathbf{f}}=\mathbf{G} \hat{\mathbf{b}} \text {. }
$$

In addition, Ó Ruanaidh and Fitzgerald (1996) show that an estimate (found by maximizing the posterior after marginalizing the amplitudes) to the Gaussian variance is

$$
\hat{\sigma}^{2}=\frac{1}{N-M}\left[\mathbf{d}^{T} \mathbf{d}-\mathbf{f}^{T} \mathbf{f}\right]
$$

This estimated variance is the data energy minus the estimated signal energy, divided by the number of degrees of freedom. Note that $\mathbf{b}$ and $\hat{\sigma}^{2}$ are functions of the analyzing frequency $[\omega]$. Equations (13) and (15) are calculated for the oscillating regions detected via Section 3 and returned as part of the results in Section 4 (see Figures 4 5, 7) and 8). 
